\title{
Moments of the Anisotropic Regularized $\kappa$-distributions
}

\author{
Klaus Scherer ${ }^{1,2}$ (1) , Marian Lazar $^{1,3}$ (1), Edin Husidic ${ }^{1}$, and Horst Fichtner ${ }^{1,2}$ (1) \\ ${ }^{1}$ Institut für Theoretische Physik, Lehrstuhl IV: Plasma-Astroteilchenphysik, Ruhr-Universität Bochum, D-44780 Bochum, Germany; kls@tp4.rub.de \\ ${ }^{2}$ Research Department, Plasmas with Complex Interactions, Ruhr-Universität Bochum, D-44780 Bochum, Germany \\ ${ }^{3}$ Centre for Mathematical Plasma Astrophysics, Celestijnenlaan 200B, B-3001 Leuven, Belgium \\ Received 2019 April 2; revised 2019 April 23; accepted 2019 April 30; published 2019 July 31
}

\begin{abstract}
For collisionless (or collision-poor) plasma populations, which are well described by the $\kappa$-distribution functions (also known as the Kappa or Lorentzian power-laws), a macroscopic interpretation has remained largely questionable, especially because of the diverging moments of these distributions. Recently significant progress has been made by introducing a generic regularization for the isotropic $\kappa$-distribution, which resolves this critical limitation. Regularization is here applied to the anisotropic forms of $\kappa$-distributions, commonly used to describe temperature anisotropies, and skewed or drifting distributions of beam-plasma systems. These regularized distributions admit nondiverging moments, which are provided for all positive $\kappa$, opening promising perspectives for a macroscopic (fluid-like) characterization of nonideal plasmas.
\end{abstract}

Key words: magnetohydrodynamics (MHD) - plasmas - solar wind

\section{Introduction}

Macroscopic models of plasmas systems are constructed on the principal (zeroth- to second-order) moments of velocity distributions of their particles. In collisionless or collision-poor plasmas, ubiquitous in space or fusion setups, the velocity distributions of charged particles are far from thermal equilibrium, exhibiting non-Maxwellian features like temperature anisotropies, beaming (or drifting) components, and suprathermal tails. Despite this evidence, theoretical predictions are still largely based on idealized scenarios assuming particles well described by bi-Maxwellian distributions, allowing for a straightforward definition of the macroscopic parameters using the main moments of the distribution. Introduced 50 yr ago by Olbert (1968) and Vasyliunas (1968) as a generalization of the idealized Maxwellian, $\kappa$-distributions have gained much notoriety in the last decades, especially for their ability to reproduce the velocity and energy distributions of plasma particles in the solar wind and planetary magnetospheres, see the review by Pierrard \& Lazar (2010). Suprathermal populations present in these environments enhance the high-energy tails of the observed distributions, which are successfully described by the $\kappa$-distribution functions. These power-laws have been widely invoked to study various kinetic effects in nonideal plasmas, e.g., the generation of suprathermal particle populations, solar wind acceleration, particle heating, wave dissipation, or instabilities as local sources of electromagnetic fluctuations. The existence of $\kappa$-distributions in space plasmas is more than obvious, but their relevance has become questionable owing to their limitations in conveying a macroscopic approach, with nondiverging moments $M_{l}$ restricted to low orders $l<2 \kappa-1$. Scherer et al. (2017) have recently introduced a regularization of $\kappa$-distributions that can resolve this limitation by removing all singularities from the theory. Introduced for simple isotropic distributions, the regularized $\kappa$ distribution (RKD)

$$
f_{R}(\kappa, w)=n_{0} N_{R}\left(1+\frac{w^{2}}{\kappa}\right)^{-\kappa-1} e^{-\alpha^{2} w^{2}}
$$

combines the standard (isotropic) $\kappa$-distribution

$$
f_{K}(\kappa, w)=n_{0} N_{K}\left(1+\frac{w^{2}}{\kappa}\right)^{-\kappa-1}
$$

with a Maxwellian cutoff $\exp \left(-\alpha^{2} w^{2}\right)$, where the regularization parameter $0<\alpha<1$ should be small enough to conform with the observations and theoretical predictions. In these expressions $w=v / \Theta$ denotes particle velocity, usually normalized to a convenient speed $\Theta, n_{0}$ is the number density of particles, and $N_{R}$ and $N_{K}$ are normalization constants, with $N_{R}$ defined in Scherer et al. (2017), Equation (9), and $N_{K}=\Gamma(\kappa) / \Gamma\left(\kappa-\frac{1}{2}\right) /\left(\sqrt{\pi^{3} \kappa} \Theta^{3}\right)$. By contrast to the standard $\kappa$-distribution, all velocity moments of the RKD are convergent for any positive $\kappa$, and have been expressed analytically in Scherer et al. (2017).

In the present paper we consider more complex distribution functions capable of reproducing kinetic anisotropies of plasma particles in collisionless plasmas from space, like, anisotropic temperatures, e.g., $A \equiv T_{\perp} / T_{\|} \neq 1$, usually defined with respect to the direction of an ambient magnetic field, or/and field-aligned beaming (or drifting) components. The anisotropic $\kappa$-models can reproduce the gyrotropic distributions of suprathermal (halo) populations measured in general in the solar wind, e.g., or bi-Kappa distribution functions (Maksimovic et al. 2005; Štverák et al. 2008), or during energetic events, like fast winds or coronal mass ejections, when the suprathermal tails of the observed distributions become skewed in the presence of field-aligned counter-moving beams (or double strahls) and resemble a product-bi-Kappa distribution, see Lazar et al. (2012). These anisotropic distribution functions are tabulated by Summers \& Thorne (1991, see their Table 1) and are employed to explain the observed fluctuations, 
Table 1

The Limits as $\kappa, \kappa_{\|}, \kappa_{\perp} \rightarrow \infty$ for the Regularized Distribution Functions

\begin{tabular}{ll}
\hline \hline$f_{\bullet}$ & $\lim _{\kappa, \kappa_{\|}, \kappa_{\perp} \rightarrow \infty} f$. \\
\hline$f_{M}$ & $n_{0} N_{M} e^{-w^{2}}$ \\
$f_{K}$ & $n_{0} N_{M} e^{-w^{2}}=f_{M}$ \\
$f_{R}$ & $n_{0} N_{R} e^{-\left(1+\alpha^{2}\right) w^{2}} \simeq n_{0} N_{M} e^{-w^{2}}=f_{M}$ \\
$f_{B K}$ & $n_{0} N_{B M} e^{-w_{\|}^{2}-w_{\perp}^{2}}=f_{B M}$ \\
$f_{\mathrm{PBK}}$ & $n_{0} N_{B M} e^{-w_{\|}^{2}-w_{\perp}^{2}}=f_{B M}$ \\
$f_{\mathrm{RBK}}$ & $n_{0} N_{\mathrm{RBK}} e^{-\left(1+\alpha_{\|}^{2}\right) w_{\|}^{2}-\left(1+\alpha_{\perp}^{2}\right) w_{\perp}^{2}} \simeq n_{0} N_{B M} e^{-w_{\|}^{2}-w_{\perp}^{2}}=f_{B M}$ \\
$f_{\mathrm{RPBK}}$ & $n_{0} N_{\mathrm{RPBK}} e^{-\left(1+\alpha_{\|}^{2}\right) w_{\|}^{2}-\left(1+\alpha_{\perp}^{2}\right) w_{\perp}^{2}} \simeq n_{0} N_{B M} e^{-w_{\|}^{2}-w_{\perp}^{2}}=f_{B M}$ \\
\hline
\end{tabular}

generated spontaneously (Viñas et al. 2015; Kim et al. 2017) or stimulated by various wave instabilities, e.g., firehose (Astfalk \& Jenko 2016; Lazar et al. 2017), cyclotron (Lazar et al. 2011; Lazar \& Poedts 2014; Lazar et al. 2015; Eliasson \& Lazar 2015; Lazar et al. 2016; dos Santos et al. 2017; Ziebell \& Gaelzer 2017; Lazar et al. 2018), or mirror instability (Leubner \& Schupfer 2002; Shaaban et al. 2018).

The regularized forms of anisotropic $\kappa$-distribution functions are introduced in Section 2, and then in Section 3 we evaluate the principal moments (zero to third order) of these distributions. The moments are calculated for distribution functions reproducing temperature anisotropies in the rest frame as well as in a drifting reference frame, in order to include anisotropic drifting components (beams, strahls, counterbeams, etc.). Potential applications are discussed in Section 4 and conclusions are formulated in Section 5. Necessary details from derivations, including mathematical definitions and symbols used, are given in the appendices. Appendix A contains a short note on the vector calculus, while some details of the moment calculations are shown in Appendix B. The evaluation of the integrals is presented in Appendix C, and finally the special treatment of the most probable speeds and heat flows is discussed in Appendix D.

\section{Regularized Anisotropic $\kappa$-distributions}

Using the same technique as in Scherer et al. (2017), here we regularize the anisotropic $\kappa$-distributions that are often used in space physics to model anisotropic temperature

$$
f_{\mathrm{RBK}}\left(\kappa, \alpha_{\|}, \alpha_{\perp}, w_{\|}, w_{\perp}\right)=n_{0} N_{\mathrm{RBK}}\left(1+\frac{w_{\|}^{2}}{\kappa}+\frac{w_{\perp}^{2}}{\kappa}\right)^{-\kappa-1} e^{-\alpha_{\|}^{2} w_{\|}^{2}-\alpha_{\perp}^{2} w_{\perp}^{2}},
$$

is the regularized bi- $\kappa(\mathrm{RBK})$ distribution function, and

$$
f_{\mathrm{RPBK}}\left(\kappa_{\|}, \kappa_{\perp}, \alpha_{\|}, \alpha_{\perp}, w_{\|}, w_{\perp}, s_{\|}, s_{\perp}\right)=n_{0} N_{\mathrm{RPBK}}\left(1+\frac{w_{\|}^{2}}{\kappa_{\|}}\right)^{-\kappa_{\|}-s_{\|}}\left(1+\frac{w_{\perp}^{2}}{\kappa_{\perp}}\right)^{-\kappa_{\perp}-s_{\perp}} e^{-\alpha_{\|}^{2} w_{\|}^{2}-\alpha_{\perp}^{2} w_{\perp}^{2}}
$$

is the regularized product-bi- $\kappa(\mathrm{RPK})$ distribution, with dimensionless velocities $w_{\|}, w_{\perp}$ (for $w$ see also above)

$$
w=\frac{v}{\Theta}, \quad w_{\|}=\frac{v_{\|}}{\Theta_{\|}}, \quad w_{\perp}=\frac{v_{\perp}}{\Theta_{\perp}} \quad \boldsymbol{w}=w_{\|} \boldsymbol{e}_{\|}+\boldsymbol{w}_{\perp},
$$

where $\boldsymbol{w}_{\perp}$ is a vector in a plane perpendicular to $\boldsymbol{e}_{\|}$. We adopt the general case with two distinct positive cutoff parameters $\alpha_{\|} \neq \alpha_{\perp}$. In the limit of $\alpha_{\|}, \alpha_{\perp} \rightarrow 0 f_{\mathrm{RBK}}$ reduces to the standard bi- $\kappa(\mathrm{BK})$ also known as bi-Kappa or bi-Lorentzian distribution function, and $f_{\mathrm{RPBK}}$ reduces to the standard product-bi- $\kappa(\mathrm{PBK})$, for $s_{\|}=1$ and $s_{\perp}=1$. Both of these standard forms are largely invoked in studies of anisotropic temperatures and their implications (see the Introduction). Notice that with $f_{\text {RPBK }}$ we have the ability to describe decoupled parallel and perpendicular components, with distinct temperatures $\left(T_{\|} \neq T_{\perp}\right)$, and distinct power indices $\left(\kappa_{\|}+s_{\|} \neq \kappa_{\perp}+s_{\perp}\right)$. We have introduced also $s_{\|}$and $s_{\perp}$ because in Lazar et al. (2012) different values for $s_{\|}$and $s_{\perp}$ are used. From the Table 3 (see below) a choice of $s_{\|}=s_{\perp}+1 / 2$ is appropriate to obtain symmetric parallel and perpendicular pressure components. For a detailed discussion, see below. In the limit of large parameters $\kappa, \kappa_{\|}, \kappa_{\perp} \rightarrow \infty$ the regularized $\kappa$ distributions approach the corresponding Maxwellian (M) and bi-Maxwellian (BM) distribution functions (see Table 1).

If $0<\alpha_{\|}, \alpha_{\perp}<1$ are small enough, Maxwellian limits of $f_{\mathrm{RPBK}}$ and $f_{\mathrm{RBK}}$ reduce both to a standard bi-Maxwellian, i.e., $f_{B M}$, with $N_{B M}=1 /\left(\pi^{3 / 2} \Theta_{\|} \Theta_{\perp}^{2}\right)$. For isotropic temperature $\left(\Theta_{\|}=\Theta_{\perp}=\Theta\right) f_{B M}$ reduces to an isotropic Maxwellian, $f_{M}$, with $N_{M}=1 /\left(\pi^{1 / 2} \Theta\right)^{3}$. In the following we do not discuss the bi- $\kappa$ distribution further, because it behaves similarly to the standard $\kappa$ distribution, concerning the poles and higher-order moments.

For drifting distributions we define a drift velocity

$$
\boldsymbol{U}_{a} \equiv \Theta_{a} \boldsymbol{W}_{a}
$$

and all moments will depend then on the dimensionless quantities $\boldsymbol{W}, W_{\|}, \boldsymbol{W}_{\perp}$ with $\boldsymbol{W}_{=}=W_{\|} \boldsymbol{e}_{\|}+\boldsymbol{W}_{\perp}$, with the appropriate normalization $\Theta, \Theta_{\|}, \Theta_{\perp}$ respectively. We neglect the argument of the moment $M$ (similar for the tensor moments), when it is zero, 
Table 2

The Moments for Nondrifting Isotropic Distribution Functions Including the Standard $\kappa$-distribution

\begin{tabular}{llll}
\hline \hline & $f_{R}$ & $f_{K}$ & $f_{M}$ \\
\hline$N$ & $(\kappa, \alpha)$ & $(\kappa)$ & $\frac{1}{\Theta^{3} \sqrt{\pi^{3}}}$ \\
\hline $\boldsymbol{u}$ & $\frac{1}{\sqrt{\pi^{3} \kappa^{3}} \Theta^{3}}\left[\mathscr{U}_{[0]}\right.$ & $\frac{\Gamma(\kappa)}{\Theta^{3} \sqrt{\pi^{3}} \sqrt{\kappa^{3}} \Gamma\left(\kappa-\frac{1}{2}\right)}$ & $\boldsymbol{0}$ \\
\hline$u_{p}$ & $\boldsymbol{0}$ & $\boldsymbol{0}$ & $\frac{2}{\sqrt{\pi}} \Theta$ \\
\hline$P_{11}$ & $\frac{2}{\sqrt{\pi}} \Theta \sqrt{\kappa}\left[{ }_{[1]} \mathscr{U}_{[0]}\right.$ & $\frac{2}{\sqrt{\pi}} \Theta \sqrt{\kappa} \frac{\Gamma(\kappa-1)}{\Gamma\left(\kappa-\frac{1}{2}\right)}$ & $\frac{1}{2} n_{0} \Theta^{2}$ \\
\hline$P_{22}=P_{33}$ & $\frac{1}{2} n_{0} \Theta^{2} \kappa_{[2]} \mathscr{U}_{[0]}$ & $n_{0} \Theta^{2} \frac{\kappa}{2 \kappa-3}$ & $P_{11, M}$ \\
\hline$p_{i j}, i \neq j$ & $P_{11, R}$ & $P_{11, K}$ & 0 \\
\hline $\boldsymbol{q}$ & 0 & 0 & $\mathbf{0}$ \\
\hline$q_{p}$ & $\mathbf{0}$ & $\mathbf{0}$ & $\frac{4}{\sqrt{\pi}} n_{0} \Theta^{3}$ \\
\hline$\overleftrightarrow{\boldsymbol{Q}}$ & $\stackrel{4}{\sqrt{\pi}} n_{0} \Theta^{3} \sqrt{\kappa^{3}}[3] \mathscr{U}_{[0]}$ & $\frac{4}{\sqrt{\pi}} n_{0} \Theta^{3} \sqrt{\kappa^{3}} \frac{\Gamma(\kappa-2)}{\Gamma\left(\kappa-\frac{1}{2}\right)}$ & $\stackrel{\leftrightarrow}{\mathbf{0}}$ \\
\hline
\end{tabular}

i.e., $M(\boldsymbol{W}=\boldsymbol{0}) \equiv M(\boldsymbol{0}) \equiv M$. This representation is particularly important in magnetized plasmas, where the magnetic field imposes a preferential direction (parallel to the magnetic field, subscript $\|$ ), leading to gyrotropic distributions, which are isotropic in the plane perpendicular (subscript $\perp$ ) to the magnetic field.

\section{Moments and Most Probable Parameters}

The moments of the distribution functions are integrals over the (entire) velocity space, where the volume elements are chosen accordingly to avoid complicated integrations. Thus, for the general moments we use Cartesian normalized volume elements $d^{3} v$, while for the normalized isotropic distribution functions $f$ spherical volume elements $w^{2} \sin \vartheta d \vartheta d \varphi d w$ are adopted, and for the anisotropic distribution functions we assume cylindrical symmetry, i.e., gyrotropic distributions typical in magnetized plasmas, with the corresponding normalized elements $w_{\perp} d w_{\|} d w_{\perp} d \vartheta$.

\subsection{General Expressions for Arbitrary Distribution Functions}

We use the following general expressions for the principal moments (of the zero, first, second, and third orders) and the most probable values defined for an arbitrary non drifting distribution function $f_{a}$

$$
\begin{gathered}
n(\boldsymbol{r}, t) \equiv M^{(0)}=\int f(\overrightarrow{\boldsymbol{r}}, \overrightarrow{\boldsymbol{v}}, t) d^{3} \boldsymbol{v} \quad \text { number density } \\
\boldsymbol{u}(\boldsymbol{r}, t) \equiv \boldsymbol{u} \equiv \boldsymbol{M}^{(1)}=\frac{1}{n(\boldsymbol{r}, t)} \int \overrightarrow{\boldsymbol{v}} f(\overrightarrow{\boldsymbol{r}}, \overrightarrow{\boldsymbol{v}}, t) d^{3} \boldsymbol{v} \quad \text { drift velocity } \\
u_{p}(\boldsymbol{r}, t) \equiv u_{p} \equiv M^{(1)}=\frac{1}{n(\boldsymbol{r}, t)} \int \boldsymbol{v} f(\overrightarrow{\boldsymbol{r}}, \overrightarrow{\boldsymbol{v}}, t) d^{3} \boldsymbol{v} \quad \text { most probable speed } \\
\overleftrightarrow{\boldsymbol{P}}(\boldsymbol{r}, t) \equiv \overleftrightarrow{\boldsymbol{P}} \equiv \stackrel{\leftrightarrow}{M}^{(2)}=\int \boldsymbol{v} \otimes \boldsymbol{v} f(\overrightarrow{\boldsymbol{r}}, \overrightarrow{\boldsymbol{v}}, t) d^{3} \boldsymbol{v} \quad \text { pressure tensor } \\
\overleftrightarrow{\boldsymbol{Q}}(\boldsymbol{r}, t) \equiv \overleftrightarrow{\boldsymbol{Q}} \equiv \overleftrightarrow{M}^{(3)}=\int \boldsymbol{v} \otimes \boldsymbol{v} \otimes \boldsymbol{v} f(\overrightarrow{\boldsymbol{r}}, \overrightarrow{\boldsymbol{v}}, t) d^{3} \boldsymbol{v} \quad \text { heat flux tensor } \\
\boldsymbol{q}(\boldsymbol{r}, t) \equiv \boldsymbol{q} \equiv \boldsymbol{M}^{(3)}=\int \boldsymbol{v}^{2} \boldsymbol{v} f(\overrightarrow{\boldsymbol{r}}, \overrightarrow{\boldsymbol{v}}, t) d^{3} \boldsymbol{v} \quad \text { heat flux vector } \\
q_{p}(\boldsymbol{r}, t) \equiv q_{p} \equiv M^{(3)}=\int \boldsymbol{v}^{3} f(\overrightarrow{\boldsymbol{r}}, \overrightarrow{\boldsymbol{v}}, t) d^{3} \boldsymbol{v} \quad \text { most probable heat flux, }
\end{gathered}
$$

where $\otimes$ is the dyadic product of vectors or tensors and $v=|v|$. If the distribution function $f$ is an even function in $w, w_{\|}, w_{\perp}$ (respectively in $v, v_{\|}, v_{\perp}$ ), the integrals involving the products with odd functions of $\boldsymbol{w}$ (or equivalently $\boldsymbol{v}$ ) vanish. Thus, without calculations one has $\boldsymbol{u}=\boldsymbol{0}$ and $\boldsymbol{q}=\boldsymbol{0}$ and $\overleftrightarrow{\boldsymbol{Q}}=\overleftrightarrow{0}$. Furthermore, in the pressure tensor only the elements in $\operatorname{tr}(\overleftrightarrow{\boldsymbol{P}}) \neq 0$, i.e., 
Table 3

The Moments for Nondrifting Distribution Functions

\begin{tabular}{|c|c|c|c|c|c|c|}
\hline & $\begin{array}{l}f_{\mathrm{PBK}} \\
\left(\kappa_{\|}, s_{\|}\right),\left(\kappa_{\perp}, s_{\perp}\right)\end{array}$ & $\begin{array}{l}f_{\mathrm{RPBK}} \\
\left(\kappa, \alpha_{\|}, s_{\|}\right),\left(\kappa, \alpha_{\perp}, s_{\perp}\right)\end{array}$ & $\begin{array}{l}f_{\mathrm{BK}} \\
(\kappa)\end{array}$ & $\begin{array}{l}f_{\mathrm{RBK}} \\
\left(\kappa, \alpha_{\|}, \alpha_{\perp}\right)\end{array}$ & $\begin{array}{l}f_{\mathrm{RBK}} \\
\left(\kappa, \alpha_{\|}=\alpha_{\perp}\right)\end{array}$ & $f_{B M}$ \\
\hline $\bar{N}$ & $\frac{\Gamma\left(\kappa_{\|}+s_{\|}\right)\left(\kappa_{\perp}+s_{\perp}-1\right)}{\sqrt{\pi^{3}} \Theta_{\|} \Theta_{\perp}^{2} \sqrt{{ }^{k} \|} \kappa_{\perp} \Gamma\left(\kappa_{\|}+s_{\|}-\frac{1}{2}\right)}$ & $\frac{\left[\mathscr{V}_{[0]}^{s \|}\left[\mathscr{V}_{[0]}^{s \perp}\right.\right.}{\sqrt{\pi^{3}} \Theta_{\|} \Theta_{\perp}^{2} \sqrt{\kappa_{\|}} \kappa_{\perp}}$ & $\frac{\Gamma(\kappa)}{\overline{\sqrt{\pi^{3} \kappa}} \Theta_{\|} \Theta^{2}{ }_{\perp}\left(\kappa-\frac{1}{2}\right)}$ & $\frac{1}{\sqrt{\pi^{3} \kappa^{3}} \Theta_{\|} \Theta_{\perp}^{2}}[] \mathscr{W}_{[0,0]}$ & $\frac{1}{\sqrt{\pi^{3} \kappa^{3}} \Theta_{\|} \Theta_{\perp}^{2}}[0] \mathscr{U}_{[]}$ & $\frac{1}{\sqrt{\pi^{3}} \Theta_{\|} \Theta_{\perp}^{2}}$ \\
\hline$u$ & 0 & 0 & 0 & 0 & 0 & 0 \\
\hline$u_{p_{\|}}$ & $\frac{1}{\sqrt{\pi}} \Theta_{\|} \frac{\sqrt{{ }^{\kappa} \|} \Gamma\left(\kappa_{\|}+s_{\|}-1\right)}{\Gamma\left(\kappa_{\|}+s_{\|}-\frac{1}{2}\right)}$ & $\frac{1}{\sqrt{\pi}} \Theta_{\| \sqrt{\kappa_{\|}}}[1]^{\mathscr{V}} \|_{[0]}$ & $2 \Theta_{\|} \sqrt{\kappa} \frac{\Gamma(\kappa-1)}{\Gamma\left(\kappa-\frac{1}{2}\right)}$ & $\frac{2}{\sqrt{\pi}} \Theta_{\|} \sqrt{\kappa} \quad{ }_{[1,0]} \mathscr{W}_{[0,0]}$ & $\frac{2}{\sqrt{\pi}} \Theta_{\|} \sqrt{\kappa} \quad[1] \mathscr{U}_{[0]}$ & $\frac{1}{\sqrt{\pi}} \Theta_{\|}$ \\
\hline$u_{p_{\perp}}$ & $\frac{\sqrt{\pi}}{2} \Theta_{\perp} \frac{\Gamma\left(\kappa_{\perp}+s_{\perp}-\frac{3}{2}\right)}{\Gamma\left(\kappa_{\perp}+s_{\perp}-1\right)}$ & $\frac{1}{2 \sqrt{\pi}} \Theta_{\perp} \sqrt{\kappa_{\perp}} \quad[1]^{\mathscr{V}_{\left[\frac{0}{0}\right]}}$ & $\frac{2}{\sqrt{\pi}} \Theta_{\perp} \sqrt{\kappa} \frac{\Gamma(\kappa-1)}{\Gamma\left(\kappa-\frac{1}{2}\right)}$ & $\frac{2}{\sqrt{\pi}} \Theta_{\perp} \sqrt{\kappa} \quad[0,1] \mathscr{W}_{[0,0]}$ & $\frac{2}{\sqrt{\pi}} \Theta_{\perp} \sqrt{\kappa} \quad{ }_{[1]} \mathscr{U}_{[0]}$ & $\frac{1}{2} \sqrt{\pi} \Theta_{\perp}$ \\
\hline$P_{11}$ & $n \Theta_{\|}^{2} \frac{\kappa \|}{2\left(\kappa \|^{+s \|)-3}\right.}$ & $\frac{1}{2} n \Theta_{\|}^{2} \kappa_{\|}[2] \mathscr{V}_{[0]}^{\|}$ & $n \Theta_{\|}^{2} \frac{\kappa}{2 \kappa-3}$ & $\frac{3}{2} n \Theta_{\|}^{2} \kappa_{[2,0]} \mathscr{W}_{[0,0]}$ & $\frac{1}{2} n \Theta_{\|}^{2} \kappa_{[2]} \mathscr{U}_{[0]}$ & $\frac{1}{2} n \Theta_{\|}^{2}$ \\
\hline$P_{22}=P_{33}$ & $n \Theta_{\perp}^{2} \frac{\kappa_{\perp}}{2\left(\kappa_{\perp}+s_{\perp}\right)-4}$ & $\frac{1}{2} n \Theta_{\perp}^{2} \kappa_{\perp[2]} \mathscr{V}_{[0]}^{\frac{1}{0}}$ & $n \Theta_{\perp}^{2} \frac{\kappa}{2 \kappa-3}$ & $\frac{3}{4} n \Theta_{\perp}^{2} \kappa[0,2] \mathscr{W}_{[0,0]}$ & $\frac{1}{2} n \Theta_{\perp}^{2} \kappa_{[2]} \mathscr{U}_{[0]}$ & $\frac{1}{2} n \Theta_{\perp}^{2}$ \\
\hline$p_{i j}, i \neq j$ & 0 & 0 & 0 & 0 & 0 & 0 \\
\hline$q$ & 0 & 0 & 0 & 0 & 0 & 0 \\
\hline$q_{p_{\|}}$ & $\frac{1}{\sqrt{\pi}} n \Theta_{\|}^{3} \sqrt{\kappa_{\|}^{3}} \frac{\Gamma\left(\kappa_{\|}+s_{\|}-2\right)}{\Gamma\left(\kappa_{\|}+s_{\|}-\frac{1}{2}\right)}$ & 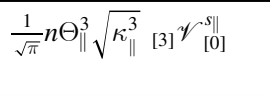 & $\frac{2}{\sqrt{\pi}} n \Theta_{\|}^{3} \sqrt{\kappa^{3}} \frac{\Gamma(\kappa-2)}{\Gamma\left(\kappa-\frac{1}{2}\right)}$ & $\frac{4}{\sqrt{\pi}} n \Theta_{\|}^{3} \sqrt{\kappa^{3}}{ }_{[3,0]} \mathscr{W}_{[0,0]}$ & $\frac{2}{\sqrt{\pi}} n \Theta_{\|}^{3} \sqrt{\kappa^{3}}[3] \mathscr{U}_{[0]}$ & $\frac{1}{\sqrt{\pi}} n \Theta_{\|}^{3}$ \\
\hline$q_{p_{\perp}}$ & $\frac{3 \sqrt{\pi}}{4} n \Theta_{\perp}^{3} \sqrt{\kappa^{3}} \frac{\Gamma\left(\kappa_{\perp}+s_{\perp}-\frac{5}{2}\right)}{\Gamma\left(\kappa_{\perp}+s_{\perp}-1\right)}$ & $\frac{3 \sqrt{\pi}}{4} n \Theta_{\perp}^{3} \sqrt{\kappa^{3}}{ }_{[3]} \mathscr{V}_{[0]}^{s_{\perp}}$ & $\frac{3 \sqrt{\pi}}{2} n \Theta_{\perp}^{3} \sqrt{\kappa^{3}} \frac{\Gamma(\kappa-2)}{2 \Gamma\left(\kappa-\frac{1}{2}\right)}$ & $\frac{3 \sqrt{\pi}}{2} n \Theta_{\perp}^{3} \sqrt{\kappa^{3}}[0,3] \mathscr{W}_{[0,0]}$ & $\frac{3 \sqrt{\pi}}{2} n \Theta_{\perp}^{3} \sqrt{\kappa^{3}}{ }_{[3]} \mathscr{U}_{[0]}$ & $\frac{3}{4} \sqrt{\pi} n \Theta_{\perp}^{3}$ \\
\hline$\overleftrightarrow{\leftrightarrow}$ & $\overleftrightarrow{0}$ & $\overleftrightarrow{\mathbf{0}}$ & $\overleftrightarrow{\mathbf{0}}$ & $\overleftrightarrow{0}$ & $\overleftrightarrow{\mathbf{0}}$ & $\overleftrightarrow{0}$ \\
\hline
\end{tabular}

Note. Column 7 Provides the Moments for the Bi-Maxwellian. 
$P_{11}, P_{22}, P_{33}$ remain. Note that, to get the correct physical units for the mass flow, pressure, and heat flow, the moments must be multiplied with the particle mass.

The situation is different when we allow for a velocity shift (i.e., a drift or bulk velocity) $\boldsymbol{U}=\Theta \boldsymbol{W} \neq 0$ in the distribution function, i.e., $f(\boldsymbol{v}-\boldsymbol{U})$. In order to evaluate the moments, in the distribution function we replace $\boldsymbol{v}-\boldsymbol{U} \rightarrow \boldsymbol{v}^{\prime}$, and, accordingly $v^{\prime 2}=(\boldsymbol{v}-\boldsymbol{U})^{2}$, and the integration variable to $\boldsymbol{v}=\boldsymbol{v}^{\prime}+\boldsymbol{U}$ and $v^{2}=\left(\boldsymbol{v}^{\prime}+\boldsymbol{U}\right)^{2}$. For the sake of simplicity we can drop "prime" and then find that $n(\boldsymbol{r}, t)$ remains unchanged, while the other moments become (with $\Theta>0$ )

$$
\begin{aligned}
\boldsymbol{u}(\boldsymbol{W}) & =\boldsymbol{U} \\
u_{p}(\boldsymbol{W}) & =\frac{1}{n(\boldsymbol{r}, t)} \int|\boldsymbol{v}+\boldsymbol{U}| f(\boldsymbol{v}, t) d^{3} \boldsymbol{v} \\
\overleftrightarrow{\boldsymbol{M}}^{(2)}(\boldsymbol{W}) & =\int(\boldsymbol{v}+\boldsymbol{U}) \otimes(\boldsymbol{v}+\boldsymbol{U}) f(\boldsymbol{v}, t) d^{3} \boldsymbol{v}=\int(\boldsymbol{v} \otimes \boldsymbol{v}+\boldsymbol{U} \otimes \boldsymbol{U}) f(\boldsymbol{v}, t) d^{3} \boldsymbol{v} \\
\stackrel{\boldsymbol{M}}{ }^{(3)}(\boldsymbol{W}) & =\int(\boldsymbol{v}+\boldsymbol{U}) \otimes(\boldsymbol{v}+\boldsymbol{U}) \otimes(\boldsymbol{v}+\boldsymbol{U}) f(\boldsymbol{v}, t) d^{3} \boldsymbol{v} \\
& =\int(\boldsymbol{v} \otimes \boldsymbol{U} \otimes \boldsymbol{v}+\boldsymbol{v} \otimes \boldsymbol{v} \otimes \boldsymbol{U}+\boldsymbol{U} \otimes \boldsymbol{v} \otimes \boldsymbol{v}+\boldsymbol{U} \otimes \boldsymbol{U} \otimes \boldsymbol{U}) f(\boldsymbol{v}, t) d^{3} v \\
\boldsymbol{q}(\boldsymbol{W}) & =\int(\boldsymbol{v}+\boldsymbol{U})^{2}(\boldsymbol{v}+\boldsymbol{U}) f(\boldsymbol{v}, t) d^{3} \boldsymbol{v}=\int\left(2(\boldsymbol{v} \cdot \boldsymbol{U}) \boldsymbol{v}+v^{2} \boldsymbol{U}+U^{2} \boldsymbol{U}\right) f(\boldsymbol{v}, t) d^{3} v \\
q_{p}(\boldsymbol{W}) & =\int|\boldsymbol{v}+\boldsymbol{U}|^{3} f(\boldsymbol{v}, t) d^{3} v,
\end{aligned}
$$

where we have neglected all terms with an even times odd function. The second-order moment can be written

$$
\stackrel{\leftrightarrow}{\boldsymbol{M}}^{(2)}=\stackrel{\leftrightarrow}{\boldsymbol{P}}+n \stackrel{\leftrightarrow}{\boldsymbol{U}}_{s}+n \stackrel{\leftrightarrow}{\boldsymbol{U}},
$$

where $\overleftrightarrow{\boldsymbol{U}}_{s}$ is a symmetric tensor, with trace $\operatorname{tr}(\stackrel{\boldsymbol{U} U}{\boldsymbol{U}})_{s}=U_{11}+U_{22}+U_{33}$ and all other elements vanish, while $\stackrel{\boldsymbol{U}}{a}$ is an antisymmetric tensor with $\operatorname{tr}(\overleftrightarrow{\boldsymbol{U} U})_{s}=0$, which describes the friction of the bulk speed. In a free flow we may neglect the latter. We may identify $n \operatorname{tr}(\overleftrightarrow{\boldsymbol{U}})_{s}$ as the "directional" ram pressure of the bulk flow. Moreover, the zeroth-, first-, second-, and third-order moment flows apparently have an analytic solution, while the moment of the most probable speed, and heat flux do not have it in general. However, if we define the most probable quantity along a parallel or perpendicular direction, an analytic solution can be found and is given below.

\subsection{Nondrifting Distributions: $\boldsymbol{W}=\mathbf{0}$}

The details of the calculation of the moments are given in the appendices, as explained below. The results are found in Table 2, where we have introduced the functions ${ }_{[m]} \mathscr{U}_{[n]}(\kappa, \alpha)$ :

$$
{ }_{[m]} \mathscr{U}_{[n]}(\kappa, \alpha)=\frac{U\left(\frac{3+m}{2}, \frac{3+m}{2}-\kappa, \alpha^{2} \kappa\right)}{U\left(\frac{3+n}{2}, \frac{3+n}{2}-\kappa, \alpha^{2} \kappa\right)} .
$$

The indices $n$ denote the numerator of the velocity integral, where the integrand is proportional to $v^{n}$, while $m$ is analogous for the denominator. The denominator is in principal the normalization, while the numerator describes the order of the moment (except for the RPBK distribution, see Appendix C for details). The function ${ }_{[m]} \mathscr{U}_{[n]}$ can be expressed as the ratio of two Kummer U (or two Tricomi) functions. Thus, for example, the pressure of the regularized $\kappa$-distribution is proportional to ${ }_{[2]} \mathscr{U}_{[0]}$ (see Table 2).

In Table 3 the moments for all nondrifting anisotropic distributions discussed above are given (for explicit calculations see Appendix C). The function ${ }_{[n, m]} \mathscr{W}_{[l, k]}\left(\kappa, \alpha_{\|}, \alpha_{\perp}\right)$ is defined in a similar way as the function ${ }_{[]} \mathscr{U}_{[]}$, but the last integral has to be solved numerically (see Equations (58), (46), and (67)).

The indices $n, m$ and $k, l$ are for the parallel and perpendicular directions respectively. For the PBK we had to introduce two slightly different functions $\left[{ }_{[m]} \mathscr{V}_{[n]}^{\|}\right.$and ${ }_{[m]} \mathscr{V}_{[n]}^{\perp}$. (Equations (47) and (48)). From these equations the moments of the other distributions (K, BK, and PBK) can easily be derived using Equations (69a), (70a), and (71b).

\subsection{Drifting Distributions: $\boldsymbol{W} \neq \boldsymbol{0}$}

The velocity and pressure moments are discussed above and are generally given by

$$
\boldsymbol{u}=\Theta \boldsymbol{W} \quad \stackrel{\leftrightarrow}{\boldsymbol{M}}(2)=\overleftrightarrow{\boldsymbol{P}}+n \overleftrightarrow{\boldsymbol{U}}
$$

Here we calculate the heat flow vector, and the most probable speeds and heat flows. The heat flow vectors for distribution functions with isotropic temperatures $(A=1)$ take the following form (see Equation (38)):

$$
\boldsymbol{q}=\left(P_{11}+n \Theta^{2} W^{2}\right) \Theta \boldsymbol{W} .
$$


Table 4

The Parameter $y_{i}$ for the Most Probable Parallel Speed

\begin{tabular}{ll}
\hline \hline & $y_{i}\left(W_{\|}\right)$ \\
\hline$u_{p, \mathrm{BM}_{\|}}$ & $\frac{W_{\|}}{\alpha_{\|}} \operatorname{erf}\left(W_{\|}\right)$ \\
$u_{p, \mathrm{RPBK}_{\|}}$ & $\frac{1}{2} W_{\|[} \mathscr{V}_{[0]}^{\|}\left(\kappa_{\|}, \alpha_{\|}\right)\{[1]$ \\
$\left.u_{p, \mathrm{RBK}_{\|}} u\left(\kappa_{\|}, \alpha_{\|}\right)+{ }_{[0]} u\left(\kappa_{\|}, \alpha_{\|}\right)\right\}$ \\
\hline
\end{tabular}

For the anisotropic temperatures we obtain (see Equation (41))

$$
\boldsymbol{q}=2\left(P_{11} \Theta_{\|} W_{\|}, P_{22} \Theta_{\perp} W_{\perp, 1}, P_{22} \Theta_{\perp} W_{\perp, 2}\right)^{T}+\left[P_{11}+P_{22}\right]\left(\Theta_{\|} W_{\|}, \Theta_{\perp} W_{\perp, 1}, \Theta_{\perp} W_{\perp, 2}\right)^{T}+n\left[\Theta_{\|}^{2} W_{\|}^{2}+\Theta_{\perp}^{2} W_{\perp}^{2}\right]\left(\Theta_{\|} W_{\|}, \Theta_{\perp} W_{\perp, 1}, \Theta_{\perp} W_{\perp, 2}\right)^{T} .
$$

The integrals are more complicated, when we treat the modulus of the velocity and the heat flow, i.e., the most probable speeds and the most probable heat flows, which are discussed in the following.

\subsubsection{The Most Probable Parallel Speed and Heat Flow}

The most probable parallel speed for anisotropic distribution functions can be written as (see Appendix D, Equations (74) and (81)):

$$
\begin{aligned}
& u_{p_{\|}}\left(W_{\|}\right)=u_{p_{\|}}(0)+\Theta_{\|} y_{i}\left(W_{\|}\right) \\
& q_{p_{\|}}\left(W_{\|}\right)=q_{p_{\|}}+3 \Theta_{\|} W_{\|} P_{2 p_{\|}}(\boldsymbol{0})+2 n \Theta_{\|}^{3} z_{i}
\end{aligned}
$$

and for the RPBK and RBK distributions $y_{i}$ and $z_{i}$ are given in Tables 4 and 5.

Case $\left|W_{\|}\right| \ll 1$-The values $y$ and $z$ can be assumed negligible for small values of $\left|W_{\|}\right| \ll 1$ and $0<\alpha_{\|} \ll 1$, implying that we can simplify $_{[n]} u$, then the required integrals have values between $\left\{0 \ldots \mid W_{\|}\right\}$and $\left|w_{\|}\right|$is also small. Thus, we may approximate the exponential in Equation (74)

$$
\left(1+\frac{w_{\|}^{2}}{\kappa}\right)^{-\kappa-1} e^{-\alpha_{\|}^{2} w^{2}} \approx\left(1+\frac{w_{\|}^{2}}{\kappa}\right)^{-\kappa-1}\left(1-\alpha_{\|}^{2} w_{\|}^{2}+\frac{\alpha_{\|}^{4} w_{\|}^{4}}{2} \ldots\right)
$$

and the leading term of the functions ${ }_{[i]} u$ can easily be calculated as

$$
\begin{aligned}
& { }_{[0]} u\left(\kappa_{\|}, \alpha_{\|}\right) \approx W_{\|_{[2]}} F_{[1]}\left(\left[\frac{1}{2}, \kappa+1\right],\left[\frac{3}{2}\right],-\frac{W_{\|}^{2}}{\kappa}\right) \ldots \\
& { }_{[1]} u\left(\kappa_{\|}, \alpha_{\|}\right) \approx \frac{1}{2}-\frac{1}{2} \kappa^{\kappa}\left(W_{\|}^{2}+\kappa\right)^{-\kappa} \ldots \\
& { }_{[2]} u\left(\kappa_{\|}, \alpha_{\|}\right) \approx W_{\|_{[2]}^{3}}^{3} F_{[1]}\left(\left[\frac{3}{2}, \kappa+1\right],\left[\frac{5}{2}\right],-\frac{W_{\|}^{2}}{\kappa}\right) \ldots \\
& { }_{[3]} u\left(\kappa_{\|}, \alpha_{\|}\right) \approx \frac{\kappa}{2(\kappa-1)}\left\{1-\kappa^{\kappa}\left(W_{\|}^{2}+1\right)\left(W_{\|}^{2}+\kappa\right)^{-\kappa}\right\} \ldots
\end{aligned}
$$

A similar approximation for the ${ }_{[i, m]} w$, leads to (see Appendix C, Equation (59))

$$
{ }_{[i, 1]} w^{\|} \approx \frac{1}{2} e^{\alpha_{\perp}^{2} \kappa} \kappa^{\kappa} \alpha_{\perp}^{2 \kappa} \Gamma\left(-\kappa, \alpha_{\perp}^{2} \kappa\right) \alpha_{\|}^{i+1}{ }_{[2]} F_{[1]}\left(\left[\frac{i+1}{2}, \kappa+1\right],\left[\frac{i+3}{2}\right],-\frac{W_{\|}^{2}}{\kappa}\right)
$$

for $i \in\{0,1,2,3\}$. The Taylor expansion for $z_{\mathrm{RPBK}}$ gives

$$
z_{\mathrm{RPBK}} \approx \frac{W_{\|}^{4}}{4} \text {. }
$$

Thus, the values $y_{\mathrm{RPBK}}, z_{\mathrm{RPBK}}$ are negligible if the parallel drift speed $W_{\|}$is low.

\subsubsection{The Perpendicular Most Probable Speed}

The most probable speeds and heat flow are calculated in Appendix D. For a constant drift velocity $\boldsymbol{W}$ the most probable speed and heat flow are given by:

$$
\begin{gathered}
u_{p_{\perp}}\left(W_{\perp}\right)=u_{p_{\perp}}(\boldsymbol{0})+n \Theta_{\|} \Theta_{\perp}^{3} W_{\perp}^{2} N \mathscr{I}_{0} \\
q_{p_{\perp}}\left(W_{\perp}\right)=q_{p_{\perp}}(\boldsymbol{0})+2 \pi \Theta_{\perp}^{2}\left[1+W_{\perp}^{2}\right] u_{p_{\perp}}(\boldsymbol{0})+n \Theta_{\|} \Theta_{\perp}^{5} W_{\perp}^{4} N \mathscr{I}_{0},
\end{gathered}
$$


Table 5

The $z_{i}$ Parameter for the Most Probable Parallel Heat Flow

\begin{tabular}{|c|c|}
\hline & $z_{i}\left(W_{\|}\right)$ \\
\hline$q_{p, \mathrm{BM}_{\|}}$ & $\frac{1}{2 \sqrt{\pi}}\left(\frac{1}{\pi \alpha_{\|}^{3}}+\frac{3 W_{\|}^{2}}{\alpha_{\|}}\right)\left(e^{-\alpha_{\|}^{2} W_{\|}^{2}}-1\right)+\frac{W_{\|} \operatorname{erf}\left(\alpha_{\|} W_{\|}\right.}{2 \pi \alpha_{\|}^{2}}\left(\frac{3}{2}+2 \alpha_{\|}^{2} W_{\|}^{2}\right)$ \\
\hline$q_{p, \mathrm{RPBK}_{\|}}$ & ${ }_{0} \mathscr{V}_{[1]}^{\|}\left(\kappa_{\|}, \alpha_{\|}\right)\left(W_{\|}^{3}[0] u\left(\kappa_{\|}, \alpha_{\|}\right)-3 W_{\|[1]}^{2} u\left(\kappa_{\|}, \alpha_{\|}\right)+3 W_{\|[2]} u\left(\kappa_{\|}, \alpha_{\|}\right)-{ }_{[3]} u\left(\kappa_{\|}, \alpha_{\|}\right)\right)$ \\
\hline$q_{p, \mathrm{RBK} \|}$ & $\begin{array}{l}{ }^{\mathscr{W}_{[0,0]}}\left(\kappa_{\|}, \kappa_{\perp}, \alpha_{\|}, \alpha_{\perp}\right)\left(W_{\|[0,0]}^{3} w\left\|\left(\kappa_{\|}, \kappa_{\perp}, \alpha_{\|}, \alpha_{\perp}\right)-3 W_{\|[1,0]}^{2} w\right\|\left(\kappa_{\|}, \kappa_{\perp}, \alpha_{\|}, \alpha_{\perp}\right)\right. \\
\left.+3 W_{\|[2,0]} w\left\|\left(\kappa_{\|}, \kappa_{\perp}, \alpha_{\|}, \alpha_{\perp}\right)-{ }_{[3,0]} w\right\|\left(\kappa_{\|}, \kappa_{\perp}, \alpha_{\|}, \alpha_{\perp}\right)\right)\end{array}$ \\
\hline
\end{tabular}

Table 6

The Most Probable Perpendicular Speed

\begin{tabular}{ll}
\hline \hline & \multicolumn{1}{l}{} \\
\hline$f_{\mathrm{BM} \perp}$ & $\frac{\sqrt{\pi^{3}}}{2 \alpha \alpha_{\perp}}$ \\
$f_{\mathrm{RPBK}_{\perp}}$ & {$\left[0 \mathscr{\mathscr { V }}_{[1]}\left(\kappa_{\perp}, \alpha_{\perp}\right)\right.$} \\
$f_{\mathrm{RBK}_{\perp}}$ & {$[0,0] \mathscr{W}_{[0,1]}\left(\kappa_{\|} \kappa_{\perp}, \alpha_{\|}, \alpha_{\perp}\right)$} \\
\hline
\end{tabular}

where $\mathscr{I}_{0}$ is given in Table 6. In the special case $\boldsymbol{W}_{w_{\perp}}=a \frac{w_{\perp}}{w_{\perp}}$ with $a \in \mathbb{R}$, we can use Equations (82) and (83) to evaluate the most probable speed and heat flow to

$$
\begin{aligned}
& u_{p_{\perp}}\left(\boldsymbol{W}_{w_{\perp}}\right)=u_{p_{\perp}}(\boldsymbol{\theta})+a \Theta_{\perp}+a^{2} \Theta_{\|} \Theta_{\perp}^{3} N \mathscr{I}_{0} \\
& q_{p_{\perp}}\left(\boldsymbol{W}_{w_{\perp}}\right)=q_{p_{\perp}}(\boldsymbol{\theta})+a \Theta_{\perp} P_{22_{\perp}}(\boldsymbol{\theta})+a^{2} \Theta_{\perp}^{2} u_{p_{\perp}}(\boldsymbol{\theta})+a^{3} n \Theta_{\perp}^{3}+a^{4} n \Theta_{\|} \Theta_{\perp}^{5} N \mathscr{I}_{0} .
\end{aligned}
$$

The above assumption about $\boldsymbol{W}_{w_{\perp}}$ can be applied for shrinking or expanding perpendicular dynamics. But a thorough discussion would go far beyond the scope of this paper. In the general case, where $\boldsymbol{w}_{\perp}=\boldsymbol{w}_{\perp 1}+\boldsymbol{w}_{\perp 2}$ no analytic solution was found (see Appendix D).

\section{Illustration and Discussion}

We have calculated in Tables 1 and 2 the pressure components $P_{11}, P_{22}, P_{33}$. Now we want to describe the components $P_{\|}$and $P_{\perp}$, i.e., the parallel and perpendicular pressure components as well as the respective temperature components. For the parallel temperature we use the classical definition, with temperature defined as the average kinetic energy

$$
k_{\mathrm{B}} T_{\|}=\frac{m}{n_{0}} \int_{-\infty}^{\infty} v_{\|}^{2} f\left(v_{\|}\right) d v_{\|}=\frac{m}{n_{0}} P_{11} \equiv \frac{m}{n_{0}} P_{\|}
$$

where we have used the decomposition $\boldsymbol{v}=v_{\|} \boldsymbol{e}_{\|}+v_{\perp, 1} \boldsymbol{e}_{\perp, 1}+v_{\perp, 2} \boldsymbol{e}_{\perp, 2}$ and the factor $1 / n_{0}$ to get the correct physical units. Here we assume that the perpendicular vectors have the same components in both directions with the unit vectors $\boldsymbol{e}_{\perp, 1,2}$. In general $\boldsymbol{v}_{\perp}$ does not need to have the same value in directions $\boldsymbol{e}_{\perp, 1}$ and $\boldsymbol{e}_{\perp, 2}$, which leads to a fully anisotropic distribution function $v_{\|} \neq v_{\perp, 1} \neq v_{\perp, 2}$, in particular, the perpendicular temperatures and pressures are also anisotropic (see Effenberger et al. 2012b, for a discussion of cosmicray diffusion). Usually, one is only interested in a decomposition of, say, the magnetic field in parallel and isotropic perpendicular components. A more general decomposition of the velocity components would also lead to a more general (R)BK, where $v_{\perp}$ has to be decomposed, then a full 3D Cartesian integration with constants $\Theta_{x}, \Theta_{y}, \Theta_{z}$ and $\alpha_{x}, \alpha_{y}, \alpha_{z}$ has to be carried out. For most purposes, it is sufficient to assume an isotropic perpendicular distribution function and use only the amplitudes $v_{\|}$and $v_{\perp}$.

This leads for the bi-Maxwellian distribution (BM) to the classical expression $k_{\mathrm{B}} T_{\|}=m \Theta_{\|}^{2} / 2$, where $\Theta_{\|}$is the classical thermal speed (see Table 2). Now defining the perpendicular temperature, one needs to be a little more careful, because the integration is now over $v_{\perp}^{2}$

$$
k_{\mathrm{B}} T_{\perp} \equiv \frac{m}{2 n_{0}} \int_{0}^{\infty}\left(v_{\perp, 1}^{2}+v_{\perp, 2}^{2}\right) f\left(v_{\perp}\right) v_{\perp} d v_{\perp}=\frac{m}{2 n_{0}}\left(P_{22}+P_{33}\right)=\frac{m}{n_{0}} P_{22}=\frac{m}{n_{0}} P_{33} \equiv \frac{m}{n_{0}} P_{\perp}
$$

the latter identities hold with the assumption of a gyrotropic distribution function (e.g., in magnetized plasmas) $P_{22}=P_{33}$.

The above result holds true for all distribution functions discussed here, even if they are not separable in the integrals discussed below. For the pressure (temperature) it turns out that the parallel and perpendicular components are mutually independent. This may hold true for more complicated distribution functions.

\subsection{Temperature Anisotropy}

We do not discuss the details of the RPBK or PBK because in the limit of $\kappa_{\|}=\kappa_{\perp}$ and $\Theta_{\|}=\Theta_{\perp}$ they do not approach the isotropic case. This is due to the fact, that when multiplying the two factors in Equation (2) (for $\kappa_{\|}+s_{\|}=\kappa_{\perp}+s_{\perp}$ ) we are always left with a 

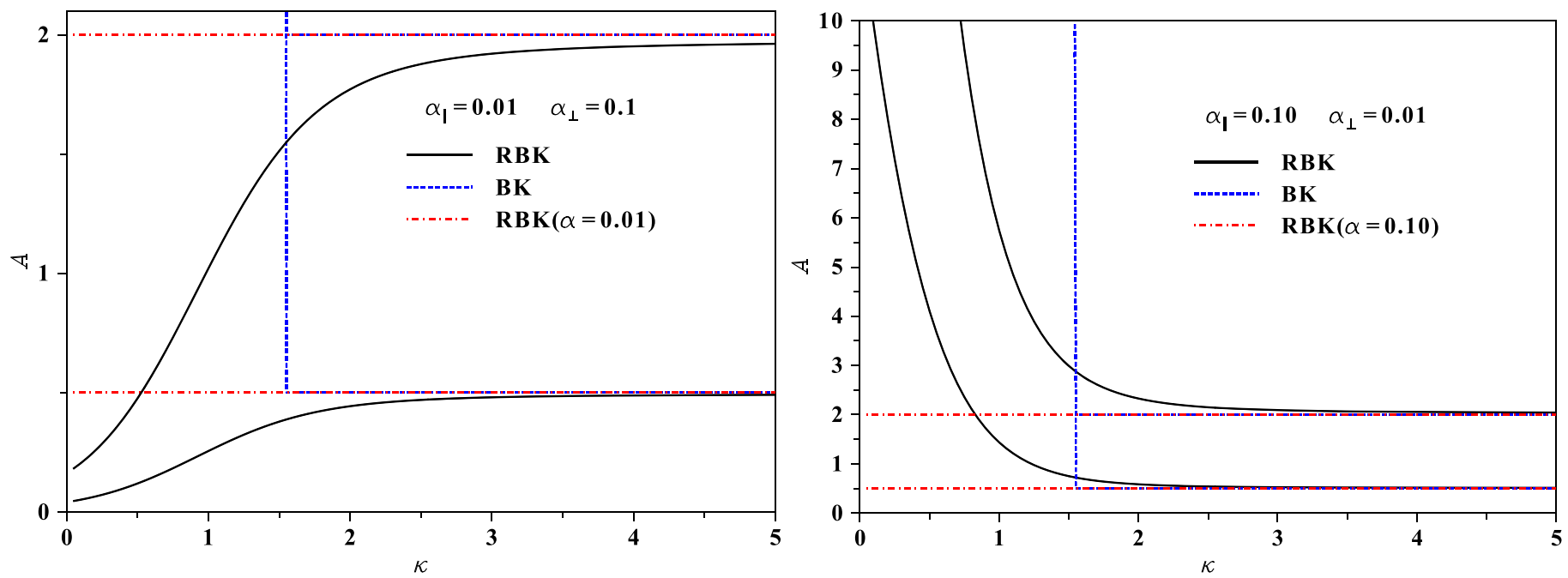

Figure 1. Anisotropy $A$. In the left panel the anisotropy for $\alpha_{\|}=0.01$ and $\alpha_{\perp}=0.1$ is shown and vice versa in the right panel. The anisotropy is chosen in such a way that for large $\kappa$ values $A=\Theta_{\perp}^{2} / \Theta_{\|}^{2}$ has a constant value, for the upper curves this is $A=2$ and for the lower curves $A=1 / 2$. For more details see the text.

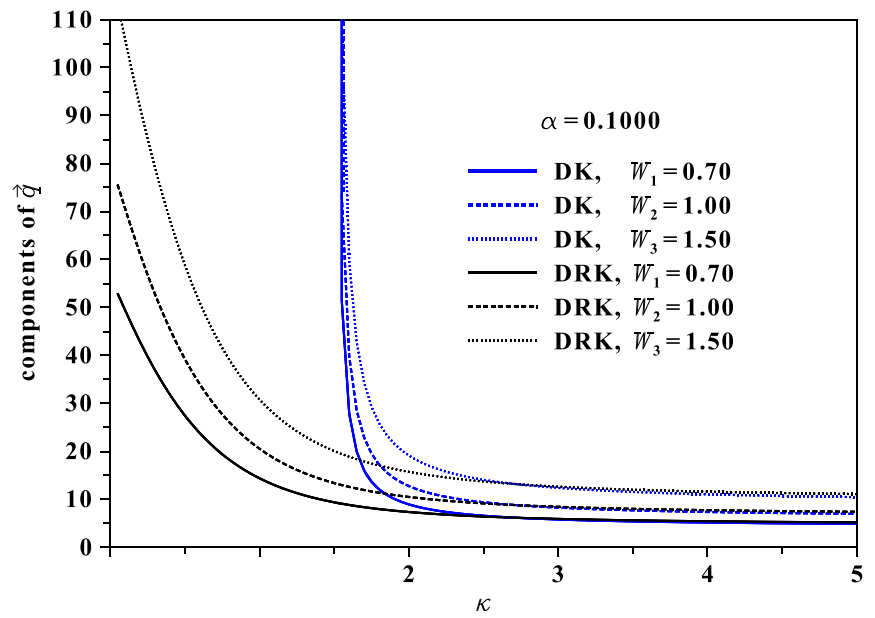

Figure 2. Heat flow for the DK- (blue) and the DRK-distributions (black) is shown for the components of the drift vector $\boldsymbol{W}=\left(W_{1}, W_{2}, W_{3}\right)^{T}$. The curves only differ by the values of the components of the drift vector $\boldsymbol{W}$. See the text for a discussion.

bi-quadratic term $v_{\|}^{2} v_{\perp}^{2}$. In the literature, often $s_{\|}=s_{\perp}=1$ is used (e.g., Lazar et al. 2012, and references therein), which leads to an asymmetric expression for the pressures $P_{11}$ and $P_{22}$, while these terms for the other discussed distribution functions (see Table 3 ) are symmetric. We can heal this behavior by choosing $s_{\|}=s_{\perp}+1 / 2$, which leads to $\kappa_{\perp}+s_{\perp}=\kappa_{\perp}+s_{\|}+1 / 2$.

In Figure 1 we have in both panels plotted the anisotropy $A=T_{\perp} / T_{\|}$which, in the limit $\kappa \rightarrow \infty$, becomes either $A=\Theta_{\perp}^{2} / \Theta_{\|}^{2}=2$ or $A=1 / 2$ for our two cases discussed in Figure 1. The red curves denote the case when in the RKB $\alpha=\alpha_{\perp}=\alpha_{\|}$, as one can see the anisotropy remains at the same value (for $A=2$ the red upper curves in both panels and for $A=1 / 2$ the lower red curves.) The $\mathrm{BK}$ is given by the blue curves, which are identical to the red ones in the range $\kappa>1.5$, below that value the BK is not defined and at $\kappa=1.5$ it is infinite. The interesting cases are the black curves, which change the anisotropy for small $\kappa<1.5$. In the left panel the cutoff parameters are $\alpha_{\|}=0.01$ and $\alpha_{\perp}=0.1$ and in the right panel $\alpha_{\|}=0.1$ and $\alpha_{\perp}=0.01$. The interesting case is that, as one can see in the left panel, the anisotropy, which is $A=2$ for large $\kappa$ values, changes its behavior from $A>1$ to $A<1$ with an intersection point $\kappa_{s}$, where $A_{s}=1$ and the anisotropy vanishes. Thus, for $A=2$ the perpendicular temperature is higher than the parallel one, both become equal at the critical point $\kappa_{s}$, and for $\kappa<\kappa_{s}$ the parallel temperature becomes higher than the perpendicular one.

In the right panel of Figure $1\left(\alpha_{\|}=0.1, \alpha_{\perp}=0.01\right)$ the anisotropy increases for small $\kappa$ values to very high values. Note that in the case of $A=1 / 2$ it becomes higher than one and, thus, the perpendicular temperature becomes higher than the parallel one. This behavior can be explained because the stronger cutoff $\alpha_{\|}>\alpha_{\perp}$ (i.e., less high speed particles) contributes to the pressure and that causes the anisotropy variation for small $\kappa$ 's, while for larger $\kappa$ values the distribution functions become more Maxwellian, and thus suppress the high speed contributions. The above described feature needs further discussions and is especially interesting for the stability of anisotropic plasmas (e.g., Shaaban et al. 2019, and references therein).

The RBK distribution for $\alpha_{\|}=\alpha_{\perp}$ (red curves) has a constant anisotropy that does not change with $\kappa$. The behavior of the BKdistribution is similar, except that anisotropy cannot be defined for $\kappa \leqslant 3 / 2$. 

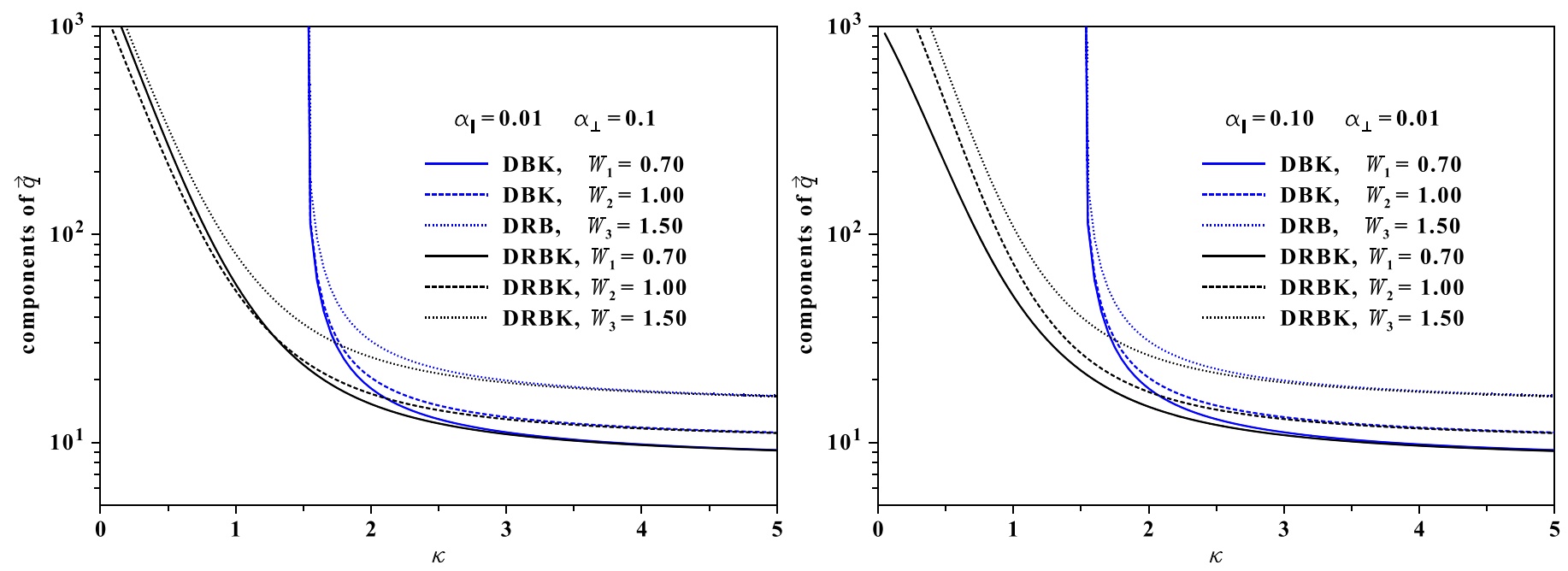

Figure 3. Similar to Figure 2 for the anisotropic heat flow for the DBK- (blue) and DRBK-distribution (black). The left panel is for the cutoff parameter $\alpha_{\|}=0.01$ and $\alpha_{\perp}=0.1$ and the lower one for $\alpha_{\|}=0.01$ and $\alpha_{\perp}=0.1$.

\subsection{The Heat Flow Vector for the Drifting RK and RBK Distributions}

In Figures 2 and 3 the heat flow is calculated for the drifting K and RK distributions (DK and DRK) as well as the BK and RBK distributions (DBK and DRBK) for the same drift vector $\boldsymbol{W}=(0.7,1,1.5)^{T}=\left(W_{\|}, W_{\perp, 1}, W_{\perp, 2}\right)^{T}$, according to Equation (11) for the isotropic and to Equation (12) for the anisotropic temperatures. In Figure 2 it can be seen that the heat flow is a constant factor for all three components (see Equation (11)) and, thus, depends strongly on the values of the drift vector $\boldsymbol{W}$ for both the DK (blue) and DRK (black) distributions.

The heat flows obtained in the case of DRBK- and DBK-distributions are shown in Figure 3 as black and blue curves, respectively. In both panels $A=1 / 2$. They have a more interesting feature: as can be seen in the left panel (for $\alpha_{\|}=0.01, \alpha_{\perp}=0.1$ ), the parallel component $W(1)$ intersects one of the perpendicular components, i.e., $W(2)$ and marginally for very small $\kappa$-values touches the $W(3)$ component. In the right panel (for $\alpha_{\|}=0.1, \alpha_{\perp}=0.01$ ) the curves do not intersect, but are obviously not parallel for small $\kappa$ values. Whether the heat flow components intersect for $\kappa<2$ depends on the choice of the drift vector components.

Thus, if $W_{\perp, 1} \neq W_{\perp, 2}$ the drift in the two perpendicular directions differs and one can expect nonisotropic turbulence or more complex diffusion tensors for cosmic-ray propagation (Effenberger et al. 2012a, 2012b). Again, this behavior needs further research and comparison with data. But that is not the goal of this work.

\section{Conclusions and Perspectives}

We have introduced new regularized forms for the anisotropic $\kappa$-distributions that can reproduce temperature anisotropies (i.e., the regularized bi- $\kappa(\mathrm{RBK})$ and the regularized product bi $-\kappa(\mathrm{PBK})$ distributions) and arbitrary drifts or flow speeds. We have shown that these distributions admit all higher-order moments, which are well defined for all values of $\kappa$. In Section 3 we have estimated these moments, e.g., the pressure and heat flux tensors, as well as the heat flow vector. For an illustration, in Section 4 we have discussed the parallel and perpendicular components of the temperature (pressure) and their anisotropies. In addition we have also estimated the heat flow components for representative drifting distributions, i.e., DRBK and DRPBK by contrast with the DBK and DPBK. The case of DRPBK is not discussed in detail because of the problems to recover the standard BK for isotropic temperatures.

For RBK and BK we discussed the general case, when the cutoff parameters $\alpha_{\|} \neq \alpha_{\perp}$ and we found that the anisotropy parameter $A$ not only depends on the ratio of the "thermal speeds" $\Theta_{\perp} / \Theta_{\|}$but also on $\kappa$ and the cutoff parameters $\left(\alpha_{\|}, \alpha_{\perp}\right)$. Interestingly, the ratio $A$ can drop from values above one to those below one, and vice versa. Also the heat flow vector for the RBK distribution shows a similar interesting feature: The heat flow components as functions of $\kappa$ can intersect for small values $(\kappa<1.5)$. The intersection point $\kappa_{s}$ depends on the components of the drift vector $\boldsymbol{W}$ (or macroscopic fluid vector). These new features can have important consequences for the interpretation of various properties of anisotropic plasmas, for example, dispersion and stability properties, which will be studied in more detail in the future.

K.S. and H.F. are grateful to the Deutsche Forschungsgemeinschaft, DFG funding the projects SCHE334/10-1 and FI706/15-1, respectively. M.L. acknowledges support from the Katholieke Universiteit Leuven, Ruhr-University Bochum, and Alexander von Humboldt Foundation. These results were obtained in the framework of the projects G0A2316N (FWO-Vlaanderen) and SCHL 201/35-1 (DFG-German Research Foundation). We also appreciate the support from the International Space Science Institute (ISSI) for hosting the international ISSI team on Kappa Distributions: From Observational Evidences via Controversial Predictions to 
a Consistent Theory of Suprathermal Space Plasmas, which triggered many fruitful discussions that were beneficial for the work presented here.

\section{Appendix A}

\section{Vector and Tensor Notation}

The dyadic and higher products are given by:

$$
\begin{aligned}
\boldsymbol{v} \otimes \boldsymbol{v} & =\stackrel{\leftrightarrow}{V}=\left(v_{i} v_{j}\right) \\
\boldsymbol{v} \otimes \boldsymbol{v} \otimes \boldsymbol{v} & =\stackrel{\leftrightarrow}{\boldsymbol{V}}=\left(\boldsymbol{v}_{i} \boldsymbol{v}_{j} \boldsymbol{v}_{k}\right)
\end{aligned}
$$

We have to distinguish between the heat flux tensor $\stackrel{\leftrightarrow}{\boldsymbol{Q}}$ and the heat flux vector $\boldsymbol{q}$ :

$$
\begin{aligned}
\overleftrightarrow{\boldsymbol{Q}} & =\int \boldsymbol{v} \otimes \boldsymbol{v} \otimes \boldsymbol{v} f d^{3} v \\
\boldsymbol{q} & =\int v^{2} \boldsymbol{v} d^{3} v
\end{aligned}
$$

The latter is also sometimes expressed as $\operatorname{tr}(\stackrel{\leftrightarrow}{Q})$.

\section{A1. Spherical Coordinates}

We define the spherical velocity vector as

$$
\boldsymbol{v}=(v \cos \varphi \sin \vartheta, v \sin \varphi \sin \vartheta, v \cos \vartheta)^{T}
$$

so that, with $w=\frac{v}{\Theta}$

$$
\Rightarrow \boldsymbol{v}=w \Theta(\cos \varphi \sin \vartheta, \sin \varphi \sin \vartheta, \cos \vartheta)^{T}
$$

and the volume element is

$$
d^{3} v=\Theta^{3} w^{2} \sin \vartheta d \vartheta d \varphi d w
$$

and the dyadic product is:

$$
\begin{aligned}
\boldsymbol{v} \otimes \boldsymbol{v} & =\overleftrightarrow{\boldsymbol{V}}=\left(v_{i} v_{j}\right)=v^{2}\left(\begin{array}{ccc}
\cos ^{2} \varphi \sin ^{2} \vartheta & \cos \varphi \sin \varphi \sin ^{2} \vartheta & \cos \varphi \sin \vartheta \cos \vartheta \\
\cos \varphi \sin \varphi \sin ^{2} \vartheta & \sin ^{2} \varphi \sin ^{2} \vartheta & \sin \varphi \sin \vartheta \cos \vartheta \\
\cos \varphi \sin \vartheta \cos \vartheta & \sin \varphi \sin \vartheta \cos \vartheta & \cos ^{2} \vartheta
\end{array}\right) \\
\boldsymbol{v} \otimes \boldsymbol{v} \otimes \boldsymbol{v} & \stackrel{\leftrightarrow}{\boldsymbol{V}}=\left(v_{i} v_{j} v_{k}\right)
\end{aligned}
$$

which is not explicitly given here, but it could be easily written out.

\section{A2. Cylindrical Coordinates}

We define

$$
\boldsymbol{v}=\left(v_{\|}, v_{\perp} \cos \vartheta, v_{\perp} \sin \vartheta\right)^{T}
$$

with

$$
w_{\|}=\frac{v_{\|}}{\Theta_{\|}}, \quad w_{\perp}=\frac{v_{\perp}}{\Theta_{\perp}} \Rightarrow \boldsymbol{v}=\left(\Theta_{\|} w_{\|}, \Theta_{\perp} w_{\perp} \cos \vartheta, \Theta_{\perp} w_{\perp} \sin \vartheta\right)^{T}
$$

and the volume element is

$$
d^{3} v=\Theta_{\|} \Theta_{\perp}^{2} w_{\perp} d w_{\|} d w_{\perp} d \vartheta
$$

and the dyadic product is:

$$
\boldsymbol{v} \otimes \boldsymbol{v}=\overleftrightarrow{\boldsymbol{V}}=\left(v_{i} v_{j}\right)=\left(\begin{array}{ccc}
v_{\|}^{2} & v_{\|} v_{\perp} \cos \vartheta & v_{\|} v_{\perp} \sin \vartheta \\
v_{\|} v_{\perp} \cos \vartheta & v_{\perp}^{2} \cos ^{2} \vartheta & v_{\perp}^{2} \sin \vartheta \cos \vartheta \\
v_{\|} v_{\perp} \sin \vartheta & v_{\perp}^{2} \sin \vartheta \cos \vartheta & v_{\perp}^{2} \sin ^{2} \vartheta
\end{array}\right)
$$




\section{Appendix B \\ Moments and Most Probable Parameter}

In the following we drop the indices of the distribution functions $f$ and normalization constants $N$. It is clear from the context which of the above presented distribution functions is meant in what follows. Note that all moments are normalized to the mass.

\section{B1. Spherical Coordinates}

In order to calculate the normalization constant $N$, we use $f^{\prime}=\frac{f}{n N}$ in the zeroth-order moment. With the isotropic volume element the moments Equation (6) is

$$
\begin{aligned}
& \frac{1}{N}=4 \pi \Theta^{3} \int_{0}^{\infty} f^{\prime} w^{2} d w \\
& u_{p}=\frac{4 \pi \Theta^{4}}{n} \int_{0}^{\infty} w^{3} f d w \\
& \stackrel{\leftrightarrow}{\boldsymbol{P}}=\Theta^{5} \int_{0}^{\infty} \int_{0}^{2 \pi} \int_{0}^{\pi}\left(\begin{array}{ccc}
\cos ^{2} \varphi \sin ^{2} \vartheta & 0 & 0 \\
0 & \sin ^{2} \varphi \sin ^{2} \vartheta & 0 \\
0 & 0 & \cos ^{2} \vartheta
\end{array}\right) f w^{4} \sin \vartheta d \vartheta d \varphi d w=\Theta^{5} \frac{4}{3} \pi\left(\begin{array}{ccc}
1 & 0 & 0 \\
0 & 1 & 0 \\
0 & 0 & 1
\end{array}\right) \int_{0}^{\infty} f w^{4} d w \\
& q_{p}=4 \pi \Theta^{6} \int_{0}^{\infty} w^{5} f d w .
\end{aligned}
$$

Only the quadratic terms in $\sin \vartheta$ and $\cos \vartheta$ survive for $\stackrel{\leftrightarrow}{P}$, and, thus, $P_{i j}=0, \forall i \neq j$.

\section{B2. Cylindrical Coordinates}

We introduce also the most probable speed $u_{p_{\|}}$along $w_{\|}$and $u_{p_{\perp}}$ along $w_{\perp}$ :

$$
\begin{aligned}
& \frac{1}{N}=\Theta_{\|} \Theta_{\perp}^{2} \int_{0}^{2 \pi} \int_{0}^{\infty} \int_{-\infty}^{\infty} f^{\prime} w_{\perp} d w_{\|} d w_{\perp} d \vartheta \\
& u_{p}=\frac{\Theta_{\|} \Theta_{\perp}^{2}}{n} \int_{0}^{2 \pi} \int_{0}^{\infty} \int_{-\infty}^{\infty} \sqrt{w_{\|}^{2} \Theta_{\|}^{2}+w_{\perp}^{2} \Theta_{\perp}^{2}} w_{\perp} f d w_{\|} d w_{\perp} d \vartheta \\
& u_{p \|}=\frac{\Theta_{\|}^{2} \Theta_{\perp}^{2}}{n} \int_{0}^{2 \pi} \int_{0}^{\infty} \int_{-\infty}^{\infty} w_{\|} w_{\perp} f d w_{\|} d w_{\perp} d \vartheta \\
& u_{p \perp}=\frac{\Theta_{\|} \Theta_{\perp}^{3}}{n} \int_{0}^{2 \pi} \int_{0}^{\infty} \int_{-\infty}^{\infty} w_{\perp}^{2} f d w_{\|} d w_{\perp} d \vartheta \\
& \overleftrightarrow{\boldsymbol{P}}=\Theta_{\|} \Theta_{\perp}^{2} \int_{0}^{2 \pi} \int_{-\infty}^{\infty} \int_{0}^{\infty}\left(\begin{array}{ccc}
w_{\|}{ }^{2} \Theta_{\|}^{2} & 0 & 0 \\
0 & w_{\perp}^{2} \Theta_{\perp}^{2} \cos ^{2} \vartheta & 0 \\
0 & 0 & w_{\perp}^{2} \Theta_{\perp}^{2} \sin ^{2} \vartheta
\end{array}\right) w_{\perp} f d w_{\|} d w_{\perp} d \vartheta \\
& =2 \pi \Theta_{\|} \Theta_{\perp}^{2} \int_{-\infty}^{\infty} \int_{0}^{\infty}\left(\begin{array}{ccc}
w_{\|}^{2} \Theta_{\|}^{2} & 0 & 0 \\
0 & \frac{1}{2} w_{\perp}^{2} \Theta_{\perp}^{2} & 0 \\
0 & 0 & \frac{1}{2} w_{\perp}^{2} \Theta_{\perp}^{2}
\end{array}\right) w_{\perp} f w_{\perp} d w_{\|} d w_{\perp} \\
& q_{p}=\Theta_{\|} \Theta_{\perp}^{2} \int_{0}^{2 \pi} \int_{0}^{\infty} \int_{-\infty}^{\infty}\left(w_{\|}^{2} \Theta_{\|}^{2}+w_{\perp}^{2} \Theta_{\perp}^{2}\right)^{3 / 2} w_{\perp} f d w_{\|} d w_{\perp} d \vartheta \\
& q_{p \|}=2 \Theta_{\|}^{4} \Theta_{\perp}^{2} \int_{0}^{2 \pi} \int_{0}^{\infty} \int_{-\infty}^{\infty} w_{\|}^{3} w_{\perp} f d w_{\|} d w_{\perp} d \vartheta \\
& q_{p \perp}=2 \Theta_{\|} \Theta_{\perp}^{5} \int_{0}^{2 \pi} \int_{0}^{\infty} \int_{-\infty}^{\infty} w_{\perp}^{4} f d w_{\|} d w_{\perp} d \vartheta .
\end{aligned}
$$

Unfortunately, the square root in the most probable parameter in Equations 34(b) and (f) does not allow, in general, for an analytic solution, as far as we know. Nevertheless, the more interesting cases are the most probable speeds along the parallel direction and in the perpendicular ones. The same holds true for the most probable heat flux.

\section{B3. With Bulk Speed $\boldsymbol{W} \neq \mathbf{0}$}

We replace $\boldsymbol{w}^{\prime} \rightarrow \boldsymbol{w}-\boldsymbol{W}, \boldsymbol{w}_{\|}^{\prime} \rightarrow \boldsymbol{w}_{\|}-\boldsymbol{W}_{\|}$, and $\boldsymbol{w}_{\perp}^{\prime} \rightarrow \boldsymbol{w}_{\perp}-\boldsymbol{W}_{\perp}$, respectively, in the distribution functions (with $W_{\|} \Theta_{\|}=U_{\|}, \ldots$ ). We discuss here only the most probable speeds, the heat flux vector, and the most probable heat flow: 


$$
\begin{aligned}
u_{p} & =\frac{\Theta}{n_{0}} \int_{0}^{\infty} \int_{0}^{2 \pi} \int_{0}^{\pi}|\boldsymbol{w}+\boldsymbol{W}|^{3} f \sin \vartheta d \vartheta d \varphi d w \\
u_{p \|} & =\frac{2 \Theta_{\|}^{2} \Theta_{\perp}^{2}}{n_{0}} \int_{0}^{2 \pi} \int_{0}^{\infty} \int_{-\infty}^{\infty}\left|w_{\|}+W_{\|}\right| w_{\perp} f d w_{\|} d w_{\perp} d \vartheta \\
u_{p \perp} & =\frac{\Theta_{\|} \Theta_{\perp}^{3}}{n_{0}} \int_{0}^{2 \pi} \int_{0}^{\infty} \int_{-\infty}^{\infty}\left(\boldsymbol{w}_{\perp}+\boldsymbol{W}_{\perp}\right)^{2} f d w_{\|} d w_{\perp} d \vartheta \\
\boldsymbol{q}_{\mathrm{I}} & =\Theta^{6} \int_{0}^{\infty} \int_{0}^{2 \pi} \int_{0}^{\pi}\left(2(\boldsymbol{w} \cdot \boldsymbol{W}) \boldsymbol{w}+w^{2} \boldsymbol{W}+W^{2} \boldsymbol{W}\right) f w^{2} \sin \vartheta d \vartheta d \varphi d w \\
\boldsymbol{q}_{\mathrm{A}} & =\Theta_{\|} \Theta_{\perp}^{2} \int_{0}^{2 \pi} \int_{0}^{\infty} \int_{-\infty}^{\infty}\left(2(\boldsymbol{v} \cdot \boldsymbol{U}) \boldsymbol{v}+v^{2} \boldsymbol{U}+U^{2} \boldsymbol{U}\right) f w_{\perp} d w_{\|} d w_{\perp} d \vartheta \\
q_{p} & =\Theta^{6} \int_{0}^{\infty} \int_{0}^{2 \pi} \int_{0}^{\pi}|\boldsymbol{w}+\boldsymbol{W}|^{5} f \sin \vartheta d \vartheta d \varphi d w \\
q_{p \|} & =\Theta_{\|}^{4} \Theta_{\perp}^{2} \int_{0}^{2 \pi} \int_{0}^{\infty} \int_{-\infty}^{\infty} \mid w_{\|}+W_{\|} \|^{3} w_{\perp} f_{\pi} d w_{\|} d w_{\perp} d \vartheta \\
q_{p \perp} & =\Theta_{\|} \Theta_{\perp}^{5} \int_{0}^{2 \pi} \int_{0}^{\infty} \int_{-\infty}^{\infty}\left(\boldsymbol{w}_{\perp}+\boldsymbol{W}_{\perp}\right)^{4} f d w_{\|} d w_{\perp} d \vartheta .
\end{aligned}
$$

For clarity, we used the following notation for the heat flow vectors: $\boldsymbol{q}_{\mathrm{I}}$ for that of the isotropic distribution functions and $\boldsymbol{q}_{\mathrm{A}}$ for that of the anisotropic ones, because they are slightly different. Additionally, we have assumed that the modulus to power $2 n$ is the same as the power $2 n:|\boldsymbol{a} \pm \boldsymbol{b}|^{2 n}=(\boldsymbol{a} \pm \boldsymbol{b})^{2 n}$. For odd powers $2 n+1$ we cannot decompose $|\boldsymbol{w} \pm \boldsymbol{W}|^{2 n+1}$, which is the case for the spherical distribution functions. But for the cylindrical parallel speeds $\mid w_{\|} \pm W_{\|}^{2 n+1}$ we can decompose the integrals into

$$
w_{\|} \pm\left. W_{\|}\right|^{2 n+1}= \begin{cases}\left(w_{\|}+W_{\|}\right)^{2 n+1} & w_{\|}, W_{\|}>0, \\ \left(w_{\|}-W_{\|}\right)^{2 n+1} & w_{\|}>W_{\|}, \\ \left(-w_{\|}+W_{\|}\right)^{2 n+1} & w_{\|}<W_{\|}, \\ \left(-w_{\|}-W_{\|}\right)^{2 n+1} & w_{\|}, W_{\|}<0 .\end{cases}
$$

These cases can then be treated separately in the integral from $-\infty$ to $\infty$, see Appendix D, where care must be taken with the integration boundaries. For the perpendicular case in cylindrical coordinates we always have a power of $2 n$ which can be decomposed. We do not calculate the heat flux tensor.

\section{B3.1. Spherical Coordinates}

The last integral of Equation (35(d)) is the heat flow along $\boldsymbol{W}$ which yields $n W^{2} \boldsymbol{W}$. The second integral is the flow of the particle energy density along $\boldsymbol{W}$ resulting in $\Theta P_{11} \boldsymbol{W}$ and the first is proportional to

$$
2 \Theta^{2} \int_{0}^{\infty} \int_{0}^{2 \pi} \int_{0}^{\pi}\left(\begin{array}{l}
w W_{1}\left(W_{1} \cos \varphi \sin \vartheta+W_{2} \sin \varphi \sin \vartheta+W_{3} v \cos \vartheta\right) \\
w W_{2}\left(W_{1} \cos \varphi \sin \vartheta+W_{2} \sin \varphi \sin \vartheta+W_{3} v \cos \vartheta\right) \\
w W_{3}\left(W_{1} \cos \varphi \sin \vartheta+W_{2} \sin \varphi \sin \vartheta+W_{3} v \cos \vartheta\right)
\end{array}\right) f w^{2} \sin \vartheta d \vartheta d \varphi d w=0
$$

and vanishes. Thus, we have for the most probable heat flow:

$$
\boldsymbol{q}=\Theta\left(P_{11}+n \Theta^{2} W^{2}\right) \boldsymbol{W}=n \Theta^{3} \boldsymbol{W}\left(\kappa_{[2]} \mathscr{U}_{[0]}(\kappa, \alpha)+W^{2}\right) .
$$

\section{B3.2. Cylindrical Coordinates}

With $\boldsymbol{W}=\left(W_{\|}, W_{\perp, 1}, W_{\perp, 2}\right)^{T}$, the first integral in Equation 35(e) gives

$$
\begin{aligned}
& 2 \Theta_{\|} \Theta_{\perp}^{2} \int_{0}^{2 \pi} \int_{0}^{\infty} \int_{-\infty}^{\infty}(\boldsymbol{v} \cdot \boldsymbol{U}) \boldsymbol{v} f w_{\perp} d w_{\|} d w_{\perp} d \vartheta=2 \Theta_{\|} \Theta_{\perp}^{2} \int_{0}^{2 \pi} \int_{0}^{\infty} \int_{-\infty}^{\infty}\left(\Theta_{\|}^{2} w_{\|} W_{\|}+\Theta_{\perp}^{2} w_{\perp} W_{\perp, 1} \cos \vartheta\right. \\
& \left.+\Theta_{\perp}^{2} w_{\perp} W_{\perp, 2} \sin \vartheta\right)\left(\begin{array}{c}
\Theta_{\|} w_{\|} \\
\Theta_{\perp} w_{\perp} \cos \vartheta \\
\Theta_{\perp} w_{\perp} \sin \vartheta
\end{array}\right) f w_{\perp} d w_{\|} d w_{\perp} d \vartheta=2 \Theta_{\|} \Theta_{\perp}^{2} \int_{0}^{\infty} \int_{-\infty}^{\infty}\left(\begin{array}{c}
2 \pi \Theta_{\|}^{3} w_{\|}^{2} w_{\perp} W_{\|} \\
\pi \Theta_{\perp}^{3} w_{\perp}^{3} W_{\perp, 1} \\
\pi \Theta_{\perp}^{3} w_{\perp}^{3} W_{\perp, 2}
\end{array}\right) f d w_{\|} d w_{\perp} \\
& \quad \mathrm{RBK} n_{\mathrm{RBK}}\left(\begin{array}{c}
\frac{3}{2} \Theta_{\|}^{3} \kappa^{\frac{3}{2}}[2,1] \mathscr{W}_{[0,0]} W_{\|} \\
\frac{3}{4} \Theta_{\perp}^{3} \kappa_{[0,3]} \mathscr{W}_{[0,0]} W_{\perp, 1} \\
\frac{3}{4} \Theta_{\perp}^{3} \kappa_{[0,3]} \mathscr{W}_{[0,0]} W_{\perp, 2}
\end{array}\right)=\kappa\left(\begin{array}{c}
P_{11} \Theta_{\|} W_{\|} \\
P_{22} \Theta_{\perp} W_{\perp, 1} \\
P_{22} \Theta_{\perp} W_{\perp, 2}
\end{array}\right)
\end{aligned}
$$


and similar with the RPBK distribution. The last integral in Equation 35(e) gives $n W^{2} \boldsymbol{W}$. The second results in

$$
\Theta_{\|} \Theta_{\perp}^{2} \int_{0}^{2 \pi} \int_{0}^{\infty} \int_{-\infty}^{\infty}\left(v_{\|}^{2}+v_{\perp}^{2}\right) f w_{\perp} d w_{\|} d w_{\perp} d \vartheta=\left(\Theta_{\|} P_{11}+\Theta_{\perp} P_{22}\right)
$$

and, thus, we have

$$
\boldsymbol{q}=2\left(\begin{array}{c}
P_{11} \Theta_{\|} W_{\|} \\
P_{22} \Theta_{\perp} W_{\perp, 1} \\
P_{22} \Theta_{\perp} W_{\perp, 2}
\end{array}\right)+\left(P_{11}+P_{22}\right)\left(\begin{array}{c}
\Theta_{\|} W_{\|} \\
\Theta_{\perp} W_{\perp, 1} \\
\Theta_{\perp} W_{\perp, 2}
\end{array}\right)+\left[n\left(\Theta_{\|}^{2} W_{\|}^{2}+\Theta_{\perp}^{2} W_{\perp}^{2}\right)\right]\left(\begin{array}{c}
\Theta_{\|} W_{\|} \\
\Theta_{\perp} W_{\perp, 1} \\
\Theta_{\perp} W_{\perp, 2}
\end{array}\right) .
$$

\section{Appendix C \\ Solutions of Integrals}

\section{C1. The Integrals of the Regularized Isotropic $\kappa$ Distribution Function}

The corresponding isotropic moments $I(\kappa, \alpha, \nu, \Theta) \equiv{ }_{[\nu]} \mathscr{U}_{[]}(\kappa, \alpha)$ of the RK distribution are (see Scherer et al. 2017)

$$
\begin{aligned}
& I(\kappa, \alpha, \nu, \Theta)=\Theta^{3+\nu} \int_{0}^{\infty}\left(1+\frac{w^{2}}{\kappa}\right)^{-\kappa-1} e^{-\alpha^{2} w^{2}} w^{2+\nu} d w=\frac{1}{2} \Theta^{3+\nu} \kappa^{\frac{3+\nu}{2}} \int_{0}^{\infty}(1+x)^{-\kappa-1} e^{-\alpha^{2} \kappa x} x^{\frac{1+\nu}{2}} d x \\
& =\frac{1}{2} \Theta^{3+\nu} \kappa^{\frac{3+\nu}{2}} \Gamma\left(\frac{3+\nu}{2}\right) U\left(\frac{3+\nu}{2}, \frac{3-2 \kappa+\nu}{2}, \alpha^{2} \kappa\right) \\
& i(\kappa, \alpha, \nu, \Theta, W)=\Theta^{3+\nu} \int_{0}^{W} w^{\nu+2}\left(1+\frac{w^{2}}{\kappa}\right)^{-\kappa-1} \mathrm{e}^{-\alpha^{2} w^{2}} d w=\frac{1}{2} \Theta^{3+\nu} \kappa^{\frac{3+\nu}{2}} \int_{0}^{W}(1+x)^{-\kappa-1} e^{-\alpha^{2} \kappa x} x^{\frac{1+\nu}{2}} d x,
\end{aligned}
$$

where $U(a, b, z)$ is the Kummer $\mathrm{U}$ or Tricomi function and $\Gamma$ is the Gamma-function, see Abramowitz \& Stegun (1972), Gradshteyn \& Ryzhik (2007), or Oldham et al. (2010). The above representation of $I(\kappa, \alpha, \nu, \Theta)$ is more compact than that in Scherer et al. (2017) and was found by Yoon et al. (2018).

Only the radial part of the spherical volume element $w^{2} \sin \vartheta d \vartheta d \varphi d w \rightarrow 4 \pi w^{2} d w$ was used, because the trigonometric part can easily be integrated.

To save writings, we define:

$$
\begin{gathered}
{ }_{[\nu]} \mathscr{U}_{[\eta]}(\kappa, \alpha) \equiv \frac{U\left(\frac{3+\nu}{2}, \frac{3-2 \kappa+\nu}{2}, \alpha^{2} \kappa\right)}{U\left(\frac{3+\eta}{2}, \frac{3-2 \kappa+\eta}{2}, \alpha^{2} \kappa\right)} \\
{ }_{[\nu]} u_{[]}(\kappa, \alpha, W) \equiv \int_{-\infty}^{W}(1+x)^{-\kappa-1} e^{-\alpha^{2} \kappa x} x^{\frac{1+\nu}{2}} d x .
\end{gathered}
$$

The first definition combines the $\kappa$ and $\alpha$ dependent parts of normalization with that from the moment $\nu$, which are dimensionless. The dimensions of the corresponding moments are given by powers of $\Theta$ and the number density $n$.

We have the following rules:

$$
\begin{aligned}
{ }_{[]} \mathscr{U}_{[\eta]} & \equiv \frac{1}{{ }_{[\eta]} \mathscr{U}_{[]}} \\
{ }_{[\nu]} \mathscr{U}_{[\eta]} & \equiv \frac{[\nu] \mathscr{U}_{[]}}{{ }_{[\eta]} \mathscr{U}_{[]}} \\
{ }_{[\nu]} \mathscr{U}_{[\eta]}{ }_{[\nu]} \mathscr{U}_{[\xi]} & ={ }_{[\nu]} \mathscr{U}_{[\xi]} \\
\frac{{ }_{[\nu]} \mathscr{U}_{[\eta]}}{{ }_{[\xi]} \mathscr{U}_{[\eta]}} & ={ }_{[\nu]} \mathscr{U}_{[\xi]} \\
{ }_{[\nu]} \mathscr{U}_{[\nu]} & =1
\end{aligned}
$$

with $\nu, \eta, \xi \in \mathbb{R}$.

So we find for the normalization constant $N_{\mathrm{RK}}$ (including a factor $2 \pi$ from the volume element) and for the elements of the moment tensors $M_{\mathrm{RK}}^{\nu}$, including the number density $n_{\mathrm{RK}}$. We do not include the factors from the integration with respect to the angle variable 
$\vartheta$ because they can differ with the order of the moments

$$
\begin{aligned}
& N_{\mathrm{RK}}=\frac{1}{\Theta^{3} \sqrt{\pi^{3} \kappa^{3}}}\left[\mathscr{U}_{[0]}\right. \\
& M_{\mathrm{RK}}^{\nu}=n_{\mathrm{RK}} N_{\mathrm{RK}} \frac{1}{2} \Theta^{3+\nu} \Gamma\left(\frac{3+\nu}{2}\right)_{[\eta]} \mathscr{U}_{[]}=n_{\mathrm{RK}} \frac{2}{\sqrt{\pi}} \Theta^{\nu} \kappa^{\frac{\nu}{2}} \Gamma\left(\frac{3+\nu}{2}\right)_{[\nu]} \mathscr{U}_{[0]} .
\end{aligned}
$$

To get the correct pressure the moment elements have to be multiplied by $4 \pi / 3$ (see Equation (33(a))).

\section{C2. The Integrals for the Product $f_{\mathrm{RPBK}}$}

For our applications, we can assume that $\lambda$ and $\mu$ have integer values for the moments of the distribution function $f_{\text {RPBK }}$ (Equation (4)) and, thus, the integral for odd values of $\lambda$ vanishes. In the case of the most probable speed and heat flow we take twice the integral $d w_{\|}$from 0 to infinity, assuming that $\left|w_{\|}\right|^{\lambda}=w_{\|}{ }^{\lambda}$. With this assumption, we find

$$
\begin{aligned}
& I\left(\kappa, \alpha_{\|}, \lambda, \Theta_{\|} I\left(\kappa, \alpha_{\perp}, \mu, \Theta_{\perp}\right)=\Theta_{\|}^{\lambda+1} \Theta_{\perp}^{\mu+2} \int_{-\infty}^{\infty} \int_{0}^{\infty}\left(1+\frac{w_{\|}^{2}}{\kappa_{\|}}\right)^{-\kappa_{\|}-s_{\|}}\left(1+\frac{w_{\perp}^{2}}{\kappa_{\perp}}\right)^{-\kappa_{\perp}-s_{\perp}} e^{-\alpha_{\|}^{2} w_{\|}^{2}-\alpha_{\perp}^{2} w_{\perp}^{2}} w_{\|}^{\lambda} w_{\perp}^{\mu+1} d w_{\|} d w_{\perp}\right. \\
& =2 \Theta_{\|}^{\lambda+1} \Theta_{\perp}^{\mu+2} \int_{0}^{\infty}\left(1+\frac{w_{\|}^{2}}{\kappa_{\|}}\right)^{-\kappa_{\|}-s_{\|}} e^{-\alpha_{\|}^{2} w_{\|}^{2}} w_{\|}^{\lambda} d w_{\|} \int_{0}^{\infty}\left(1+\frac{w_{\perp}^{2}}{\kappa_{\perp}}\right)^{-\kappa_{\perp}-s_{\perp}} e^{-\alpha_{\perp}^{2} w_{\perp}^{2}} w_{\perp}^{\mu+1} d w_{\perp} \\
& =\frac{1}{2} \Theta_{\|}^{\lambda+1} \Theta_{\perp}^{\mu+2} \kappa_{\|}^{\frac{\lambda+1}{2}} \frac{\mu+2}{\kappa_{\perp}^{2}}\left(\int(1+x)^{-\kappa_{\|}-s_{\|}} e^{-\alpha_{\|}^{2} \kappa_{\|} x} x^{\frac{\lambda-1}{2}} d x\right)\left(\int(1+x)^{-\kappa_{\perp}-s_{\perp}} e^{-\alpha_{\perp}^{2} \kappa_{\perp} x} x^{\frac{\mu}{2}} d x\right) \\
& =\frac{1}{2} \Theta_{\|}^{\lambda+1} \Theta_{\perp}^{\mu+2} \kappa_{\|}^{\frac{\lambda+1}{2}} \frac{\mu+2}{\kappa_{\perp}^{2}} \Gamma\left(\frac{\lambda+1}{2}\right) \Gamma\left(\frac{\mu+2}{2}\right)_{[\lambda]} \mathscr{V}_{[]}^{\|}\left(\kappa_{\|}, \alpha_{\|}\right)_{[\mu]} \mathscr{V}_{\frac{\perp}{\perp}}\left(\kappa_{\perp}, \alpha_{\perp}\right),
\end{aligned}
$$

where we have defined

$$
\begin{aligned}
& { }_{[\lambda]} \mathscr{V}_{[]}^{\|}\left(\kappa_{\|}, \alpha_{\|}\right) \equiv U\left(\frac{\lambda+1}{2}, \frac{\lambda+3}{2}-\kappa_{\|}-s_{\|}, \kappa_{\|} \alpha_{\|}^{2}\right) \\
& {[\mu]^{\mathscr{V}_{\perp}}\left(\kappa_{\perp}, \alpha_{\perp}\right) \equiv U\left(\frac{\mu+2}{2}, \frac{\mu+4}{2}-\kappa_{\perp}-s_{\perp}, \kappa_{\perp} \alpha_{\perp}^{2}\right) \text {. }}
\end{aligned}
$$

For $i \in\{\|, \perp\}$ we define analogously as above:

$$
{ }_{[x]} \mathscr{V}_{[y]}^{i}\left(\kappa_{i}, \alpha_{i}\right)=\frac{{ }_{[x]} \mathscr{V}_{[]}^{i}}{{ }_{[]^{\mathscr{V}}} \mathscr{V}_{[y]}^{i}}\left(\kappa_{i}, \alpha_{i}\right)={ }_{[x]} \mathscr{V}_{[y]}^{i}=\frac{{ }_{[x]} \mathscr{V}_{[]}^{i}}{{ }_{[]^{\mathscr{V}}}^{i}} .
$$

Finally, we get for the normalization constant and the elements of the moment tensors (see above Equations (45) with a factor $2 \pi$ from the cylindrical volume element)

$$
\begin{aligned}
N_{\mathrm{RPBK}}^{\|, \perp} & =\frac{1}{\sqrt{\pi^{3}} \Theta_{\|} \Theta_{\perp}^{2} \kappa_{\|}^{\frac{1}{2}} \kappa_{\perp}}\left[{ }^{\mathscr{V}}{ }_{[0]}^{\|}\left(\kappa_{\|}, \alpha_{\|}\right)_{[} \mathscr{V}_{[0]}^{\perp}\left(\kappa_{\perp}, \alpha_{\perp}\right)\right. \\
M_{\mathrm{RPBK}}^{\lambda, \mu, s_{\|}, S_{\perp}} & =n_{\mathrm{RPBK}} N_{\mathrm{RPBK}}^{S \mid, S_{\perp}} \frac{1}{2} \Theta_{\|}^{\lambda+1} \Theta_{\perp}^{\mu+2} \kappa_{\|}^{\frac{\lambda+1}{2}} \frac{\mu+2}{\kappa^{2}} \Gamma\left(\frac{\lambda+1}{2}\right) \Gamma\left(\frac{\mu+2}{2}\right)_{[\lambda]} \mathscr{V}_{[]}^{\|}\left(\kappa_{\|}, \alpha_{\|}\right)_{[\mu]} \mathscr{V}_{[]}\left(\kappa_{\perp} \alpha_{\perp}\right) \Pi \\
& =n_{\mathrm{RPBK}} \frac{1}{2 \sqrt{\pi^{3}}} \Theta_{\|}^{\lambda} \Theta_{\perp}^{\mu} \kappa_{\|}^{\frac{\lambda}{2}} \kappa_{\perp}^{\frac{\mu}{2}} \Gamma\left(\frac{\lambda+1}{2}\right) \Gamma\left(\frac{\mu+2}{2}\right)_{[\lambda]} \mathscr{V}_{[0]}^{\|}\left(\kappa_{\|}, \alpha_{\|}\right)_{[\mu]} \mathscr{V}_{[0]}^{\perp}\left(\kappa_{\perp}, \alpha_{\perp}\right) \Pi .
\end{aligned}
$$

To get the correct pressure elements $\left(P_{11}, P_{22}=P_{33}\right)$ we have to multiply the above moment elements by the factors $\Pi$ as calculated in the matrix Equation 34(e)

$$
\Pi= \begin{cases}2 \pi & \text { for } P_{11} \\ \pi & \text { for } P_{22} \text { and } P_{33} .\end{cases}
$$

Similar scaling factors have to be calculated for higher-order moments, which are not discussed here. For the most probable speeds and heat flows a factor $2 \pi$ as in the normalization constant $N_{\mathrm{RPBK}}$ needs to be multiplied. 


\section{C3. The Moments of $f_{\mathrm{RBK}}$}

The $f_{\mathrm{RBK}}$ is given by

$$
f_{\mathrm{RBK}}=f_{\mathrm{RBK}}\left(\kappa, \alpha_{\|}, \alpha_{\perp}, \Theta_{\|}, \Theta_{\perp}, v_{\|}, v_{\perp}\right)=n_{0} N_{\mathrm{RBK}}\left(1+\frac{v_{\|}^{2}}{\kappa \Theta_{\|}^{2}}+\frac{v_{\perp}^{2}}{\kappa \Theta_{\perp}^{2}}\right)^{-\kappa-1} e^{-\frac{\alpha_{\Pi}^{2} v_{\|}^{2}}{\Theta_{\|}^{2}}-\frac{\alpha_{\perp}^{2} v_{\perp}^{2}}{\Theta_{\perp}^{2}}} .
$$

Thus, we have to solve the integrals below, where we do not take into account the integration with respect to $\vartheta$, which is straightforward using the equations from Appendix A. To save writings we define: $J\left(\kappa, \alpha_{\|}, \alpha_{\perp}, \lambda, \mu, \Theta_{\|}, \Theta_{\perp}\right) \equiv J$ and $j\left\|\left(\kappa, \alpha_{\|}, \alpha_{\perp}, \lambda, \mu, \Theta_{\|}, \Theta_{\perp}\right) \equiv j\right\|$ and, analogously, for the perpendicular direction

$$
\begin{aligned}
& J=\Theta_{\|}^{1+\lambda} \Theta_{\perp}^{2+\mu} \int_{-\infty}^{\infty} \int_{0}^{\infty} w_{\|}^{\lambda} w_{\perp}^{\mu+1}\left(1+\frac{w_{\|}^{2}}{\kappa}+\frac{w_{\perp}^{2}}{\kappa}\right)^{-\kappa-1} e^{-\alpha_{\|}^{2} w_{\|}{ }^{2}-\alpha_{\perp}^{2} w_{\perp}^{2}} d w_{\perp} d w_{\|} \\
& j \|=\Theta_{\|}^{1+\lambda} \Theta_{\perp}^{2+\mu} \int_{-\infty}^{W_{\|}} \int_{0}^{\infty} w_{\|}^{\lambda} w_{\perp}^{\mu+1}\left(1+\frac{w_{\|}^{2}}{\kappa}+\frac{w_{\perp}^{2}}{\kappa}\right)^{-\kappa-1} e^{-\alpha_{\|}^{2} w_{\|}{ }^{2}-\alpha_{\perp}^{2} w_{\perp}^{2}} d w_{\perp} d w_{\|} \\
& j^{\perp}=\Theta_{\|}^{1+\lambda} \Theta_{\perp}^{2+\mu} \int_{-\infty}^{\infty} \int_{0}^{W_{\perp}} w_{\|}^{\lambda} w_{\perp}^{\mu+1}\left(1+\frac{w_{\|}^{2}}{\kappa}+\frac{w_{\perp}^{2}}{\kappa}\right)^{-\kappa-1} e^{-\alpha_{\|}^{2} w_{\|}{ }^{2}-\alpha_{\perp}^{2} w_{\perp}^{2}} d w_{\perp} d w_{\|} .
\end{aligned}
$$

With the following substitution:

$$
\begin{aligned}
& w_{\perp}=r \sin \vartheta \quad w_{\|}=r \cos \vartheta, \quad r=\sqrt{w_{\perp}^{2}+w_{\|}^{2}} \\
& J=\Theta_{\|}^{1+\lambda} \Theta_{\perp}^{2+\mu} \int_{0}^{\pi} \int_{0}^{\infty} r^{\lambda+\mu+2}\left(1+\frac{r^{2}}{\kappa}\right)^{-\kappa-1} e^{-r^{2}\left(\alpha_{\perp}^{2}+\left(\alpha_{\|}^{2}-\alpha_{\perp}^{2}\right) \cos ^{2} \vartheta\right)} \sin ^{\mu+1} \vartheta \cos ^{\lambda} \vartheta d r d \vartheta .
\end{aligned}
$$

The integral with respect to $r$ is similar to $I(\kappa, \alpha, \nu, \Theta)$. Thus, we have to solve the following type of integral (with $a^{2}=\alpha_{\|}^{2}-\alpha_{\perp}^{2}$ ), which we take twice from 0 to $\frac{\pi}{2}$ to account for the cases when we want to calculate the most probable parameters:

$$
\begin{aligned}
& J=\Theta_{\|}^{1+\lambda} \Theta_{\perp}^{2+\mu} \kappa^{3+\lambda+\mu} \int_{0}^{\frac{\pi}{2}} \int_{0}^{\infty}(1+x)^{-\kappa-1} e^{-\alpha_{\perp}^{2} \kappa x} e^{-\kappa a^{2} x \cos ^{2} \vartheta} x^{\frac{1+\lambda+\mu}{2}} \sin ^{\mu+1} \vartheta \cos ^{\lambda} \vartheta d x d \vartheta \\
& =\Theta_{\|}^{1+\lambda} \Theta_{\perp}^{2+\mu} \kappa^{3+\lambda+\mu} \int_{0}^{1} \int_{0}^{\infty}(1+x)^{-\kappa-1} e^{-\alpha_{\perp}^{2} \kappa x} e^{-\kappa a^{2} x t^{2}} x^{\frac{1+\lambda+\mu}{2}}\left(1-t^{2}\right)^{\frac{\mu}{2}} t^{\lambda} d x d t \\
& =\Theta_{\|}^{1+\lambda} \Theta_{\perp}^{2+\mu} \kappa^{3+\lambda+\mu} \Gamma\left(\frac{3+\lambda+\mu}{2}\right) \int_{0}^{1} U\left(\frac{3+\lambda+\mu}{2}, \frac{3+\lambda+\mu}{2}-\kappa, \kappa\left[\alpha_{\perp}^{2}+\left(\alpha_{\|}^{2}-\alpha_{\perp}^{2}\right) t^{2}\right]\right)\left(1-t^{2}\right)^{\frac{\mu}{2}} t^{\lambda} d t .
\end{aligned}
$$

The integral cannot be solved in general and a numeric solution is required. Nevertheless, we can define

$$
\begin{aligned}
{ }_{[\lambda, \mu]} \mathscr{W}_{[]}\left(\kappa, \alpha_{\|}, \alpha_{\perp}\right) & \equiv \int_{0}^{1} U\left(\frac{3+\lambda+\mu}{2}, \frac{3+\lambda+\mu}{2}-\kappa, \kappa\left[\alpha_{\perp}^{2}+\left(\alpha_{\|}^{2}-\alpha_{\perp}^{2}\right) t^{2}\right]\right)\left(1-t^{2}\right)^{\frac{\mu}{2}} t^{\lambda} d t \\
{ }_{[\lambda, \mu]}{ }^{\|} \|\left(\kappa, \alpha_{\|}, \alpha_{\perp}, W_{\|}\right) & \equiv \int_{-\infty}^{W_{\|}} \int_{0}^{\infty} w_{\|}^{\lambda} w_{\perp}^{\mu+1}\left(1+\frac{w_{\|}^{2}}{\kappa}+\frac{w_{\perp}^{2}}{\kappa}\right)^{-\kappa-1} e^{-\alpha_{\|}^{2} w_{\|}{ }^{2}-\alpha_{\perp}^{2} w_{\perp}^{2}} d w_{\perp} d w_{\|} \\
{[\lambda, \mu]^{w}{ }^{\perp}\left(\kappa, \alpha_{\|}, \alpha_{\perp}, W_{\perp}\right) } & \equiv \int_{-\infty}^{W_{\perp}} \int_{0}^{\infty} w_{\|}^{\lambda} w_{\perp}^{\mu+1}\left(1+\frac{w_{\|}^{2}}{\kappa}+\frac{w_{\perp}^{2}}{\kappa}\right)^{-\kappa-1} e^{-\alpha_{\|}^{2} w_{\|}^{2}-\alpha_{\perp}^{2} w_{\perp}^{2} d w_{\perp} d w_{\|}}
\end{aligned}
$$

and solve the remaining integral numerically. If $\alpha_{\|}=\alpha_{\perp} \equiv \alpha$ the above integral reduces to

$$
[\lambda, \alpha] \mathscr{W}_{[]}(\kappa, \alpha, \alpha)=\frac{1}{2} \frac{\Gamma\left(\frac{1+\lambda}{2}\right) \Gamma\left(\frac{2+\mu}{2}\right)}{\Gamma\left(\frac{3+\lambda+\mu}{2}\right)}[\lambda+\mu] \mathscr{U}_{[]}(\kappa, \alpha) .
$$

The above solution still depends on the perpendicular and parallel values $\Theta_{\|}$and $\Theta_{\perp}$ and the power indices $\lambda$, $\mu$ in the $\Gamma$ functions: $\Gamma\left(\frac{1+\lambda}{2}\right)$ and $\Gamma\left(\frac{2+\mu}{2}\right)$ 
We have the following identities:

$$
\begin{aligned}
{ }_{[\lambda, \mu]} \mathscr{W}_{[]} & ={ }_{[]} \mathscr{W}_{[\lambda, \mu]}^{-1} \\
{ }_{[\lambda, \mu]} \mathscr{W}_{[\nu, \eta]} & \equiv{ }_{[\lambda, \mu]} \mathscr{W}_{[][]} \mathscr{W}_{[\nu, \eta]} \\
{[\lambda, \mu] } & \mathscr{W}_{[\lambda, \mu]}=1 \\
{[\lambda, \mu] } & \mathscr{W}_{[]} \neq{ }_{[\mu, \lambda]} \mathscr{W}_{[]} .
\end{aligned}
$$

Again we find for the normalization and tensor elements:

1. for $\alpha_{\|} \neq \alpha_{\perp}$

$$
\begin{aligned}
& N_{\mathrm{RBK}}=\frac{1}{\sqrt{\pi^{3}} \Theta_{\|} \Theta_{\perp}^{2} \sqrt{\kappa^{3}}}\left[\mathscr{W}_{[0,0]}\left(\kappa, \alpha_{\perp}\right)\right. \\
& M_{\mathrm{RBK}}^{\lambda, \mu}=n_{\mathrm{RBK}} N_{\mathrm{RBK}} \Theta_{\|}^{\lambda+1} \Theta_{\perp}^{\mu+2} \kappa \kappa^{\frac{\lambda+\mu+3}{2}} \Gamma\left(\frac{3+\lambda+\mu}{2}\right){ }_{[\lambda, \mu]} \mathscr{W}_{[]}\left(\kappa, \alpha_{\|}, \alpha_{\perp}\right) \Pi \\
& =n_{\mathrm{RBK}} \frac{1}{\sqrt{\pi^{3}}} \Theta_{\|}^{\lambda} \Theta_{\perp}^{\mu} \kappa^{\frac{\lambda+\mu}{2}} \Gamma\left(\frac{3+\lambda+\mu}{2}\right){ }_{[\lambda, \mu]} \mathscr{W}_{0,0}\left(\kappa, \alpha_{\|}, \alpha_{\perp}\right) \Pi .
\end{aligned}
$$

2. for $\alpha_{\|}=\alpha_{\perp}=\alpha$

$$
\begin{aligned}
N_{\mathrm{RPK}} & =\frac{1}{\sqrt{\pi^{3}} \Theta_{\|} \Theta_{\perp}^{2} \sqrt{\kappa^{3}}}\left[\mathscr{U}_{[0]}(\kappa, \alpha)\right. \\
M_{\mathrm{RBK}}^{\lambda, \mu} & =n_{\mathrm{RBK}} N_{\mathrm{RBK}} \Theta_{\|}^{\lambda+1} \Theta_{\perp}^{\mu+2} \kappa^{\frac{\lambda+\mu+3}{2}} \Gamma\left(\frac{\lambda+1}{1}\right) \Gamma\left(\frac{\mu+2}{2}\right)_{[\lambda+\mu]} \mathscr{U}_{[]} \Pi \\
& =n_{\mathrm{RBK}} \frac{1}{\sqrt{\pi^{3}}} \Theta_{\|}^{\lambda} \Theta_{\perp}^{\mu} \kappa^{\frac{\lambda+\mu}{2}} \Gamma\left(\frac{\lambda+1}{2}\right) \Gamma\left(\frac{\mu+2}{2}\right){ }_{[\lambda+\mu]} \mathscr{U}_{[0]}(\kappa, \alpha) \Pi
\end{aligned}
$$

and for the pressure elements $\Pi$ is given Equation (51).

\section{C4. The Moments of the Distribution Functions $f_{K}, f_{B M}$, and $f_{P B M}$}

The moments of the distribution functions $f_{K}$, and $f_{\mathrm{BK}}$ can be obtained setting $\alpha=\alpha_{\|}=\alpha_{\perp}$ in Equation 42(a) and Equation (61), where care must be taken, because the second argument of the Tricomi function should be lower than one:

$$
\begin{aligned}
& \lim _{\substack{\alpha^{2} \kappa \rightarrow 0 \\
\frac{3+\nu}{2}-\kappa<1}} U\left(\frac{3+\nu}{2}, \frac{3+\nu}{2}-\kappa, \alpha^{2} \kappa\right)=\frac{\Gamma\left(\kappa-\frac{1+\nu}{2}\right)}{\Gamma(\kappa+1)} \\
& \lim _{\substack{\alpha \kappa_{\|}^{2} \kappa_{\| \rightarrow 0} \\
\frac{3+\lambda}{2}-\kappa-s_{\|}<1}}[\lambda]^{\mathscr{V}^{s_{\|}}}=\lim _{\substack{\alpha_{\|}^{2} \kappa_{\| \rightarrow 0} \rightarrow \\
\frac{3+\lambda}{2}-\kappa_{\|}-s_{\|}<1}} U\left(\left(\frac{1+\lambda}{2}, \frac{3+\lambda}{2}-\kappa_{\|}-s_{\|}\right)=\frac{\Gamma\left(\kappa_{\|}+s_{\|}-\frac{\lambda+1}{2}\right)}{\Gamma\left(\kappa_{\|}+s_{\perp}\right)}\right. \\
& \lim _{\substack{\alpha_{\perp}^{2} \kappa_{\perp} \rightarrow 0 \\
\frac{\mu+4}{2}-\kappa_{\perp}-s_{\perp}<1}}[\mu]^{\mathscr{V}_{[]}^{s_{\perp}}}=\lim _{\substack{\alpha_{\perp}^{2} \kappa_{\| \rightarrow 0} \rightarrow \\
\frac{\mu+4}{2}-\kappa_{\perp}-s_{\perp}<1}} U\left(\left(\frac{\mu+2}{2}, \frac{\mu+4}{2}-\kappa_{\perp}-s_{\perp}\right)=\frac{\Gamma\left(\kappa_{\perp}+s_{\perp}-\frac{\mu+2}{2}\right)}{\Gamma\left(\kappa_{\perp}+s_{\perp}\right)} .\right.
\end{aligned}
$$

Note that the factors for the integration over the angle variable are only included in the normalization, and have to be handled for the tensor elements as above. Thus, the moments for $f_{K}$ are:

$$
\begin{gathered}
N_{K}=\frac{2 \Gamma(\kappa+1)}{\Theta^{3} \sqrt{\pi^{3} \kappa^{3}} \Gamma\left(\kappa-\frac{1}{2}\right)} \\
M_{K}^{(\nu)}=N_{K} \Theta^{\nu+3} \kappa^{\frac{3+\nu}{2}} \Gamma\left(\frac{3+\nu}{2}\right) \frac{\Gamma\left(\kappa-\frac{1+\nu}{2}\right)}{\Gamma(\kappa+1)}=\frac{1}{\sqrt{\pi}} \Theta^{\nu} \kappa^{\frac{\nu}{2}} \Gamma\left(\frac{3+\nu}{2}\right) \frac{\Gamma\left(\kappa-\frac{1+\nu}{2}\right)}{\Gamma\left(\kappa-\frac{1}{2}\right)}
\end{gathered}
$$


and those for $f_{B K}$ :

$$
\begin{aligned}
N_{B K} & =\frac{\Gamma(\kappa+1)}{\sqrt{\pi^{3} \kappa^{3}} \Theta_{\|} \Theta_{\perp}^{2} \Gamma\left(\kappa-\frac{1}{2}\right)} \\
M_{B K}^{(\lambda, \mu)} & =\frac{N_{B K}}{\sqrt{\pi^{3}}} \Theta_{\|}^{1+\lambda} \Theta_{\perp}^{2+\mu} \kappa^{\frac{3+\lambda+\mu}{2}} \frac{\Gamma\left(\frac{1+\lambda}{2}\right) \Gamma\left(\frac{2+\mu}{2}\right) \Gamma\left(\kappa-\frac{1+\lambda+\mu}{2}\right)}{\Gamma(\kappa+1)} \Pi \\
& =\frac{1}{2 \sqrt{\pi^{3}}} \Theta_{\|}^{\lambda} \Theta_{\perp}^{\mu} \kappa^{\frac{\lambda+\mu}{2}} \frac{\Gamma\left(\frac{1+\lambda}{2}\right) \Gamma\left(\frac{2+\mu}{2}\right) \Gamma\left(\kappa-\frac{1+\lambda+\mu}{2}\right)}{\Gamma\left(\kappa-\frac{1}{2}\right)} \Pi .
\end{aligned}
$$

If $\Theta_{\|}=\Theta_{\perp}$ we get the values for $N_{K}$ and $M_{K}^{(\nu)}$ with $\lambda+\mu=\nu$, except for $\Gamma\left(\frac{1+\lambda}{2}\right) \Gamma\left(\frac{2+\mu}{2}\right) \neq \Gamma\left(\frac{3+\nu}{2}\right)$, but $\lambda=\nu+2$ and $\mu=0$.

For the $f_{P B K}$ distribution we must be a little more careful, because of the product, but nevertheless we can use the limiting approach from Equation 46:

$$
\begin{aligned}
N_{\mathrm{PBK}} & =\frac{\Gamma\left(\kappa_{\|}+s_{\|}\right) \Gamma\left(\kappa_{\perp}+s_{\perp}\right)}{\sqrt{\pi^{3}} \sqrt{\kappa_{\|}} \kappa_{\perp} \Theta_{\|} \Theta_{\perp}^{2} \Gamma\left(\kappa_{\|}+s_{\|}-\frac{1}{2}\right) \Gamma\left(\kappa_{\perp}+s_{\perp}-1\right)} \\
M_{\mathrm{PBK}}^{(\lambda, \mu)} & =n_{\mathrm{PBK}} N_{\mathrm{PBK}} \frac{1}{2} \Theta_{\|}^{\lambda+1} \Theta_{\perp}^{\mu+2} \kappa_{\|}^{\frac{\lambda+1}{2}} \frac{\mu+2}{\kappa_{\perp}^{2}} \Gamma\left(\frac{\lambda+1}{2}\right) \Gamma\left(\frac{\mu+2}{2}\right) \frac{\Gamma\left(\kappa_{\|}+s_{\|}-\frac{\lambda+1}{2}\right)}{\Gamma\left(\kappa_{\|}+s_{\|}\right)} \frac{\Gamma\left(\kappa_{\perp}+s_{\perp}-\frac{\mu+2}{2}\right)}{\Gamma\left(\kappa_{\perp}+s_{\perp}\right)} \Pi \\
& =n_{\mathrm{PBK}} \frac{\Theta_{\|}^{\lambda} \Theta_{\perp}^{\mu}}{2 \sqrt{\pi^{3}}} \kappa_{\|}^{\frac{\lambda}{2}} \kappa_{\perp}^{\frac{\mu}{2}} \Gamma\left(\frac{\lambda+1}{2}\right) \Gamma\left(\frac{\mu+2}{2}\right) \frac{\Gamma\left(\kappa_{\|}+s_{\|}-\frac{\lambda+1}{2}\right) \Gamma\left(\kappa_{\perp}+s_{\perp}-\frac{\mu+2}{2}\right)}{\Gamma\left(\kappa_{\|}+s_{\|}-\frac{1}{2}\right) \Gamma\left(\kappa_{\perp}+s_{\perp}-1\right)} \Pi .
\end{aligned}
$$

In the limit $\Theta_{\|}=\Theta_{\perp}$ and $\kappa_{\|}=\kappa_{\perp}$ the isotropic case seems to be completely different, because of the different $\Gamma$-functions in Equation (71b).

The moments for the isotropic case are found in Table 2 and that for the anisotropic distributions in Table 3.

The condition $\frac{3+\nu}{2}-\kappa<1$ is reflected in those for the standard $\kappa$-distributions, whose moments diverge below those values.

\section{Appendix D}

The Most Probable Parameters, $u_{p}, u_{p_{\|}}, u_{p_{\perp}}, q_{p}, q_{p_{\|}}, q_{p_{\perp}}$

$$
\text { D1. With } W_{\|}=0, W_{\perp}=0
$$

We process the integrals as follows (with $\tilde{f}\left(w_{\|}, w_{\perp}\right) \equiv f\left(w_{\|}, w_{\perp}, \alpha_{\|}, \alpha_{\perp}\right) /(n N)$ and dropping the indices):

$$
\begin{aligned}
& \frac{1}{\Theta_{\|}^{2} \Theta_{\perp}^{2} N} u_{p_{\|}}\left(w_{\|}=0\right)=\int_{0}^{2 \pi} \int_{0}^{\infty} \int_{-\infty}^{\infty} \mid w_{\|} \| \tilde{f}\left(w_{\|}, w_{\perp}\right) w_{\perp} d w_{\|} d w_{\perp} d \vartheta \\
& \quad=\int_{0}^{2 \pi} \int_{0}^{\infty} \int_{-\infty}^{0}\left|w_{\|}\right| \tilde{f}\left(w_{\|}, w_{\perp}\right) w_{\perp} d w_{\|} d w_{\perp} d \vartheta+\int_{0}^{2 \pi} \int_{0}^{\infty} \int_{0}^{\infty} w_{\|} \tilde{f}\left(w_{\|}, w_{\perp}\right) w_{\perp} d w_{\|} d w_{\perp} d \vartheta \\
& =\int_{0}^{2 \pi} \int_{0}^{\infty} \int_{\infty}^{0} \mid-w_{\|} \tilde{f}\left(-w_{\|}, w_{\perp}\right) w_{\perp} d\left(-w_{\|} d w_{\perp} d \vartheta+\int_{0}^{2 \pi} \int_{0}^{\infty} \int_{0}^{\infty} w_{\|} \tilde{f}\left(w_{\|}, w_{\perp}\right) w_{\perp} d w_{\|} d w_{\perp} d \vartheta\right. \\
& =2 \int_{0}^{2 \pi} \int_{0}^{\infty} \int_{0}^{\infty} w_{\|} \tilde{f}\left(w_{\|}, w_{\perp}\right) w_{\perp} d w_{\|} d w_{\perp} d \vartheta
\end{aligned}
$$

where we have substituted in the first integral of the third line $w_{\|} \rightarrow-w_{\|}$and then changing the integral boundaries leads to the fourth line, because $f\left(-w_{\|}, w_{\perp}\right)=f\left(w_{\|}, w_{\perp}\right)$. For the most probable heat flow $q_{p, \pi \|}$ we find analogously:

$$
\begin{aligned}
& \frac{1}{n \Theta_{\|}^{4} \Theta_{\perp}^{2} N} q_{p_{\|}}\left(W_{\|}=0\right)=\int_{0}^{2 \pi} \int_{0}^{\infty} \int_{-\infty}^{\infty} \mid w_{\|} \|^{3} \tilde{f}\left(w_{\|}, w_{\perp}\right) w_{\perp} d w_{\|} d w_{\perp} d \vartheta \\
& =2 \int_{0}^{2 \pi} \int_{0}^{\infty} \int_{0}^{\infty} w_{\|}^{3} \tilde{f}\left(w_{\|}, w_{\perp}\right) w_{\perp} d w_{\|} d w_{\perp} d \vartheta .
\end{aligned}
$$




\section{D2. With $W_{\|} \neq 0, \boldsymbol{W}_{\perp}=\mathbf{0}$}

\section{D2.1. The Most Probable Speed}

Now with a positive shift $W_{\|}>0$ in the distribution function

$$
\begin{gathered}
\frac{1}{\Theta_{\|}^{2} \Theta_{\perp}^{2} N} u_{p_{\|}}\left(W_{\|} \neq 0\right)=\int_{0}^{2 \pi} \int_{0}^{\infty} \int_{-\infty}^{\infty}\left|w_{\|}\right| \tilde{f}\left(w_{\|}-W_{\|}, w_{\perp}\right) w_{\perp} d w_{\|} d w_{\perp} d \vartheta \\
=\int_{0}^{2 \pi} \int_{-\infty}^{\infty} \int_{-\infty}^{0}\left|w_{\|}\right| \tilde{f}\left(w_{\|}^{\prime}, w_{\perp}\right) w_{\perp} d w_{\|} d w_{\perp} d \vartheta \quad \text { with } \quad w_{\|}^{\prime}=w_{\|}+W_{\|} \quad \text { and } \quad w_{\|}^{\prime}=w_{\|}-W_{\|} \\
=\int_{0}^{2 \pi} \int_{0}^{\infty} \int_{-\infty}^{\infty} \mid w_{\|}^{\prime}+W_{\|} \tilde{f}\left(w_{\|}^{\prime}, w_{\perp}\right) w_{\perp} d w_{\|}^{\prime} d w_{\perp} d \vartheta \\
=\int_{0}^{2 \pi} \int_{0}^{\infty} \int_{-\infty}^{0}\left|w_{\|}^{\prime}+W_{\|} \tilde{f}\left(w_{\|}^{\prime}, w_{\perp}\right) w_{\perp} d w_{\|}^{\prime} d w_{\perp} d \vartheta+\int_{0}^{2 \pi} \int_{0}^{\infty} \int_{0}^{\infty}\right| w_{\|}^{\prime}+W_{\|} \tilde{f}\left(w_{\|}^{\prime}, w_{\perp}\right) w_{\perp} d w_{\|}^{\prime} d w_{\perp} d \vartheta
\end{gathered}
$$

Now we replace $w_{\|}^{\prime}=-w_{\|}$and replace the modulus according to the decomposition Equation $(36)$ by $\mid W_{\|}-\tilde{w}_{\|} \rightarrow\left(W_{\|}-\tilde{w}_{\|}\right)$for $W_{\|}>\tilde{w}_{\|}$and $\mid W_{\|}-\tilde{w}_{\|} \rightarrow\left(\tilde{w}_{\|}-W_{\|}\right)$for $W_{\|}<\tilde{w}_{\|}$in the first integral, while the last is expressed as $u_{\pi, p_{\|}}(0)$ and $W_{\|}$

$$
\begin{aligned}
u_{p_{\|}}= & \Theta_{\|}^{2} \Theta_{\perp}^{2} N \int_{0}^{2 \pi} \int_{0}^{\infty} \int_{W_{\|}}^{\infty}\left(w_{\|}-W_{\|}\right) \tilde{f}\left(w_{\|}, w_{\perp}\right) w_{\perp} d w_{\|} d w_{\perp} d \vartheta \\
& +\Theta_{\|}^{2} \Theta_{\perp}^{2} N \int_{0}^{2 \pi} \int_{0}^{\infty} \int_{0}^{W_{\|}}\left(W_{\|}-w_{\|}\right) \tilde{f}\left(w_{\|}, w_{\perp}\right) w_{\perp} d w_{\|} d w_{\perp} d \vartheta+\frac{1}{2} u_{\pi, p_{\|}}(0)+\frac{1}{2} \Theta_{\|} W_{\|} \\
= & \Theta_{\|}^{2} \Theta_{\perp}^{2} N \int_{0}^{2 \pi} \int_{0}^{\infty} \int_{0}^{W_{\|}}\left(w_{\|}-W_{\|}\right) \tilde{f}\left(w_{\|}, w_{\perp}\right) w_{\perp} d w_{\|} d w_{\perp} d \vartheta \\
& -\Theta_{\|}^{2} \Theta_{\perp}^{2} N \int_{0}^{2 \pi} \int_{0}^{\infty} \int_{0}^{W_{\|}}\left(W_{\|}-w_{\|}\right) \tilde{f}\left(w_{\|}, w_{\perp}\right) w_{\perp} d w_{\|} d w_{\perp} d \vartheta+u_{\pi, p_{\|}}(0) \\
= & 2 \Theta_{\|}^{2} \Theta_{\perp}^{2} N \int_{0}^{2 \pi} \int_{0}^{\infty} \int_{0}^{W_{\|}}\left(W_{\|}-w_{\|}\right) \tilde{f}\left(w_{\|}, w_{\perp}\right) w_{\perp} d w_{\|} d w_{\perp} d \vartheta+u_{\pi, p_{\|}}(0) .
\end{aligned}
$$

When $W_{\|}$is close to zero, the integral vanishes and we are left with $u_{p_{\|}}(0)$, while when $W_{\|}$becomes large, we can assume that the term $u_{\pi, p_{\|}}(0)$ cancels with that in the integral, and we are left with $W_{\|} \Theta_{\|}$.

\section{D2.2. The Most Probable Heat Flow}

We proceed again analogously to Equation (72) (assuming $W_{\|}<0$ ) and find

$$
\begin{aligned}
& \frac{1}{n \Theta_{\|}^{4} \Theta_{\perp}^{2} N} q_{p_{\|}}\left(W_{\|} \neq 0\right)=\int_{0}^{2 \pi} \int_{0}^{\infty} \int_{-\infty}^{\infty} \mid w_{\|}^{3} \tilde{f}\left(w_{\|}-W_{\|}, w_{\perp}\right) w_{\perp} d w_{\|} d w_{\perp} d \vartheta \\
& =\int_{0}^{2 \pi} \int_{0}^{\infty} \int_{-\infty}^{0}\left|w_{\|}^{\prime}+W_{\|}{ }^{3} \tilde{f}\left(w_{\|}^{\prime}, w_{\perp}\right) w_{\perp} d w_{\|}^{\prime} d w_{\perp} d \vartheta+\int_{0}^{2 \pi} \int_{0}^{\infty} \int_{0}^{\infty}\right| w_{\|}^{\prime}+W_{\|}{ }^{3} \tilde{f}\left(w_{\|}^{\prime}, w_{\perp}\right) w_{\perp} d w_{\|}^{\prime} d w_{\perp} d \vartheta \\
& =\mathscr{I}_{1}+\mathscr{I}_{2} .
\end{aligned}
$$

We replace $\left|w_{\|}^{\prime}+W_{\|}\right|^{3}$ by $\left(w_{\|}^{\prime}+W_{\|}\right)^{3}$ in the second integral $\mathscr{I}_{2}$, and in the first integral $\mathscr{I}_{1}$ we apply the decomposition (36)

$$
\left|w_{\|}^{\prime}+W_{\|}\right|^{3}= \begin{cases}\left(W_{\|}-w_{\|}^{\prime}\right)^{3} & W_{\|}>w_{\|}^{\prime} \\ \left(w_{\|}^{\prime}-W_{\|}\right)^{3} & W_{\|}<w_{\|}^{\prime},\end{cases}
$$

Then the second integral $\mathscr{I}_{2}$ gives (neglecting the primes):

$$
\begin{aligned}
& n \Theta_{\|}^{4} \Theta_{\perp}^{2} N \mathscr{I}_{2}=n \Theta_{\|}^{4} \Theta_{\perp}^{2} N \int_{0}^{2 \pi} \int_{0}^{\infty} \int_{0}^{\infty}\left(w_{\|}+W_{\|}\right)^{3} \tilde{f}\left(w_{\|}, w_{\perp}\right) w_{\perp} d w_{\|} d w_{\perp} d \vartheta \\
& =n \Theta_{\|}^{4} \Theta_{\perp}^{2} N \int_{0}^{2 \pi} \int_{0}^{\infty} \int_{0}^{\infty}\left(w_{\|}^{3}+3 w_{\|}^{2} W_{\|}+3 w_{\|} W_{\|}^{2}+W_{\|}^{3}\right) \tilde{f}\left(w_{\|}, w_{\perp}\right) w_{\perp} d w_{\|} d w_{\perp} d \vartheta \\
& =\frac{1}{2} q_{p, \Pi_{\|}}(0)+\frac{3}{2} \Theta_{\|} W_{\|} P_{11, \Pi}(0)+\frac{3}{2} \Theta_{\|}^{2} W_{\|}^{2} u_{p, \Pi \|}(0)+\pi n \Theta_{\|}^{4} \Theta_{\perp}^{2} N_{\|} W_{\|}^{3} \int_{0}^{\infty} \int_{0}^{\infty} \tilde{f}\left(w_{\|}, w_{\perp}\right) d w_{\|} d w_{\perp} d \vartheta
\end{aligned}
$$


The first integral $\mathscr{I}_{1}$ gives (changing $w^{\prime} \rightarrow-w$ ):

$$
\begin{aligned}
n \Theta_{\|}^{4} \Theta_{\perp}^{2} N \mathscr{I}_{2} & =n \Theta_{\|}^{4} \Theta_{\perp}^{2} N \int_{0}^{2 \pi} \int_{0}^{\infty} \int_{0}^{\infty} \mid-w_{\|}+W_{\|^{3}}^{3} \tilde{f}\left(w_{\|}, w_{\perp}\right) w_{\perp} d w_{\|} d w_{\perp} d \vartheta \\
& =n \Theta_{\|}^{4} \Theta_{\perp}^{2} N \int_{0}^{2 \pi} \int_{0}^{\infty} \int_{0}^{\infty} \mid-w_{\|}+W_{\|}^{3} \tilde{f}\left(w_{\|}, w_{\perp}\right) w_{\perp} d w_{\|} d w_{\perp} d \vartheta \\
& =n \Theta_{\|}^{4} \Theta_{\perp}^{2} N \int_{0}^{2 \pi} \int_{0}^{\infty} \int_{0}^{W_{\|}}\left(W_{\|}-w_{\|}\right)^{3} \tilde{f}\left(w_{\|}, w_{\perp}\right) w_{\perp} d w_{\|} d w_{\perp} d \vartheta \\
& +n \Theta_{\|}^{4} \Theta_{\perp}^{2} N \int_{0}^{2 \pi} \int_{0}^{\infty} \int_{W_{\|}}^{\infty}\left(w_{\|}-W_{\|}\right)^{3} \tilde{f}\left(w_{\|}, w_{\perp}\right) w_{\perp} d w_{\|} d w_{\perp} d \vartheta
\end{aligned}
$$

In the second integral we change the lower boundary $W_{\|}$to zero and subtract the integral from zero to $W_{\|}$, then we get finally

$$
\begin{gathered}
q_{p, \Pi_{\|}}\left(W_{\|}\right)=2 n N \int_{0}^{2 \pi} \int_{0}^{\infty} \int_{0}^{W_{\|}}\left(W_{\|}-w_{\|}\right)^{3} \tilde{f}\left(w_{\|}, w_{\perp}\right) w_{\perp} d w_{\|} d w_{\perp} d \vartheta+q_{p, \Pi_{\|}}(0)+3 \Theta_{\|} P_{22 p, \Pi_{\|}}(0) . \\
\text { D3. With } W_{\|}=0, \boldsymbol{W}_{\perp} \neq \boldsymbol{0}
\end{gathered}
$$

We assume first that $\boldsymbol{W}_{\|}=$const. For the integrals, containing $|\boldsymbol{w}+\boldsymbol{W}|$ or $\left|\boldsymbol{w}_{\perp}+\boldsymbol{W}_{\perp}\right|^{2 n+1}$, no analytic solution could be found, but in the anisotropic case we have the form $\left|\boldsymbol{w}_{\perp}+\boldsymbol{W}_{\perp}\right|^{2 n}=\left(\boldsymbol{w}_{\perp}+\boldsymbol{W}_{\perp}\right)^{2 n}$, with $\boldsymbol{W}_{\perp}=W_{1_{\perp}} \boldsymbol{e}_{1_{\perp}}+W_{2_{\perp}} \boldsymbol{e}_{2_{\perp}}$. We need the integrals containing $n=1$ and $n=2$, for $n=1$ we find

$$
\begin{aligned}
\left(\boldsymbol{w}_{\perp}+\boldsymbol{W}_{\perp}\right)^{2} & =w_{\perp}^{2}+2 \boldsymbol{w}_{\perp} \boldsymbol{W}_{\perp}+W_{\perp}^{2} \\
& =w_{\perp}^{2}+2\left(w_{\perp} W_{1_{\perp}} \cos \vartheta+w_{\perp} W_{2_{\perp}} \sin \vartheta\right)+W_{\perp}^{2} .
\end{aligned}
$$

The integration over a full period of $\cos \vartheta, \sin \vartheta$ vanishes and we are left only with the squares $w_{\perp}^{2}, W_{\perp}^{2}$. Similar for $n=2$

$$
\begin{aligned}
\left(\boldsymbol{w}_{\perp}+\boldsymbol{W}_{\perp}\right)^{4} & =w_{\perp}^{4}+4 w_{\perp}^{2} \boldsymbol{w}_{\perp} \boldsymbol{W}_{\perp}+4 W_{\perp}^{2} \boldsymbol{w}_{\perp} \boldsymbol{W}_{\perp}+2 w_{\perp}^{2} W_{\perp}^{2}+W_{\perp}^{4}+4\left(\boldsymbol{w}_{\perp} \boldsymbol{W}_{\perp}\right)^{2} \\
& =w_{\perp}^{4}+W_{\perp}^{4}+2 w_{\perp}^{2} W_{\perp}^{2}+4\left(w_{\perp}^{2} W_{1_{\perp}}^{2} \cos ^{2} \vartheta+w_{\perp}^{2} W_{2_{\perp}}^{2} \sin ^{2} \vartheta\right),
\end{aligned}
$$

where we have dropped in the last line all terms including $\cos \vartheta$, $\sin \vartheta$ or $\cos \vartheta \sin \vartheta$.

Thus, the most probable speed and heat flow are given by:

$$
\begin{gathered}
u_{p_{\perp}}\left(W_{\perp}\right)=n \Theta_{\|} \Theta_{\perp}^{3} N \int_{0}^{2 \pi} \int_{-\infty}^{\infty} \int_{0}^{\infty}\left(w_{\perp}^{2}+W_{\perp}^{2}\right) \tilde{f}\left(w_{\|}, w_{\perp}\right) d w_{\|} d w_{\perp} d \vartheta \\
=u_{p, \Pi_{\perp}}(\mathbf{0})+n \Theta_{\|} \Theta_{\perp}^{3} N \mathscr{I}_{0} W_{\perp}^{2} \\
q_{p_{\perp}}=n \Theta_{\|} \Theta_{\perp}^{5} N \int_{0}^{2 \pi} \int_{-\infty}^{\infty} \int_{0}^{\infty}\left(w_{\perp}^{4}+W_{\perp}^{4}+2 w_{\perp}^{2} W_{\perp}^{2}+4\left(w_{\perp}^{2} W_{1_{\perp}}^{2} \cos ^{2} \vartheta+w_{\perp}^{2} W_{2_{\perp}}^{2} \sin ^{2} \vartheta\right) \tilde{f}\left(w_{\|}, w_{\perp}\right) d w_{\perp} d w_{\|} d \vartheta\right. \\
=q_{p_{\perp}}(\mathbf{0})+2 \pi \Theta_{\perp}^{2}\left[1+W_{\perp}^{2}\right] u_{p_{\perp}}(\mathbf{0})+n \Theta_{\|} \Theta_{\perp}^{5} N \mathscr{I}_{0} W_{\perp}^{4}
\end{gathered}
$$

with

$$
\mathscr{I}_{0}=\int_{0}^{2 \pi} \int_{0}^{\infty} \int_{-\infty}^{\infty} f\left(w_{\|}, w_{\perp}\right) d w_{\|} d w_{\perp} d \vartheta .
$$

If we have the special case where $\boldsymbol{W}_{w_{\perp}} \| \boldsymbol{w}_{\perp}$ or $\boldsymbol{W}_{w_{\perp}}=a \frac{\boldsymbol{w}_{\perp}}{w_{\perp}}$ with $a \in \mathbb{R}$, we can use Equations (82) and (83) to evaluate the most probable speed and heat flow. Thus, we have:

$$
\begin{aligned}
& u_{p_{\perp}}\left(\boldsymbol{W}_{w_{\perp}}\right)=u_{p, \Pi_{\perp}}(\boldsymbol{0})+a \Theta_{\perp}+a^{2} n \Theta_{\|} \Theta_{\perp}^{3} N \mathscr{I}_{0} \\
& q_{p_{\perp}}\left(\boldsymbol{W}_{w_{\perp}}\right)=q_{p, \Pi_{\perp}}(\boldsymbol{0})+a \Theta_{\perp} P_{22 \pi, s e}(\boldsymbol{0})+a^{2} \Theta_{\perp}^{2} u_{p, \Pi_{\perp}}(\boldsymbol{0})+a^{3} n \Theta_{\perp}^{3}+a^{4} n \Theta_{\|} \Theta_{\perp}^{5} N \mathscr{I}_{0} .
\end{aligned}
$$

The values for $\mathscr{I}_{0}$ are given in Table 6 in the main text.

\section{ORCID iDs}

Klaus Scherer (1) https://orcid.org/0000-0002-9530-1396

Marian Lazar (iD https://orcid.org/0000-0002-8508-5466

Horst Fichtner (iD https://orcid.org/0000-0002-9151-5127 


\section{References}

Abramowitz, M., \& Stegun, I. A. 1972, Handbook of Mathematical Functions (New York: Dover)

Astfalk, P., \& Jenko, F. 2016, JGRA, 121, 2842

dos Santos, M. S., Ziebell, L. F., \& Gaelzer, R. 2017, Ap\&SS, 362, 18

Effenberger, F., Fichtner, H., Scherer, K., et al. 2012a, ApJ, 750, 108

Effenberger, F., Fichtner, H., Scherer, K., \& Büsching, I. 2012b, A\&A, 547, A120

Eliasson, B., \& Lazar, M. 2015, PhPl, 22, 062109

Gradshteyn, I. S., \& Ryzhik, I. M. 2007, Table of Integrals, Series, and Products (New York: Elsevier)

Kim, S., Schlickeiser, R., Yoon, P. H., López, R. A., \& Lazar, M. 2017, PPCF, 59, 125003

Lazar, M., Fichtner, H., \& Yoon, P. H. 2016, A\&A, 589, A39

Lazar, M., Pierrard, V., Poedts, S., \& Schlickeiser, R. 2012, ASSP, 33, 97

Lazar, M., \& Poedts, S. 2014, MNRAS, 437, 641

Lazar, M., Poedts, S., \& Fichtner, H. 2015, A\&A, 582, A124

Lazar, M., Poedts, S., \& Schlickeiser, R. 2011, MNRAS, 410, 663

Lazar, M., Shaaban, S. M., Poedts, S., \& Štverák, Š. 2017, MNRAS, 464, 564

Lazar, M., Yoon, P. H., López, R. A., \& Moya, P. S. 2018, JGRA, 123, 6
Leubner, M. P., \& Schupfer, N. 2002, NPGeo, 9, 75

Maksimovic, M., Zouganelis, I., Chaufray, J.-Y., et al. 2005, JGRA, 110 , A09104

Olbert, S. 1968, in Physics of the Magnetosphere, Vol. 10, ed. R. D. L. Carovillano \& J. F. McClay (Dordrecht: Reidel), 641

Oldham, K., Myland, J., \& Spanier, J. 2010, An Atlas of Functions: with Equator, the Atlas Function Calculator, An Atlas of Functions (New York: Springer)

Pierrard, V., \& Lazar, M. 2010, SoPh, 267, 153

Scherer, K., Fichtner, H., \& Lazar, M. 2017, EL, 120, 50002

Shaaban, S. M., Lazar, M., Astfalk, P., \& Poedts, S. 2018, JGRA, 123, 1754

Shaaban, S. M., Lazar, M., López, R. A., Fichtner, H., \& Poedts, S. 2019, MNRAS, 483, 5642

Štverák, Š., Trávníček, P., Maksimovic, M., et al. 2008, JGRA, 113, A03103

Summers, D., \& Thorne, R. M. 1991, PhFlB, 3, 1835

Vasyliunas, V. M. 1968, in Physics of the Magnetosphere, Vol. 10, ed. R. D. L. Carovillano \& J. F. McClay (Dordrecht: Reidel), 622

Viñas, A. F., Moya, P. S., Navarro, R. E., et al. 2015, JGRA, 120, 3307

Yoon, P. H., Lazar, M., Scherer, K., Fichtner, H., \& Schlickeiser, R. 2018, ApJ, 868,131

Ziebell, L. F., \& Gaelzer, R. 2017, PhPl, 24, 102108 